\title{
High-accurate SPH method with Multidimensional Optimal Order Detection limiting
}

\author{
Xesús Nogueira, Luis Ramírez, Stéphane Clain, Raphaël Loubère, \\ Luis Cueto-Felgueroso, Ignasi Colominas
}

\section{Highlights}

- A new high-accurate SPH method based on Riemann solvers is presented.

- MLS approximations are used for the reconstruction step in the Riemann solver.

- The stability of the scheme is achieved by the a posteriori MOOD paradigm.

- Important gains in accuracy are obtained for problems involving non-smooth flows.

\begin{abstract}
We present a new high-accurate, stable and low-dissipative Smooth Particle Hydrodynamics (SPH) method based on Riemann solvers. The method derives from the SPH-ALE formulation first p roposed b y Vila a nd B en M oussa. M oving L east Squares approximations are used for the reconstruction of the variables and the computation of Taylor expansions. The stability of the scheme is achieved by the a posteriori Multi-dimensional Optimal Order Detection (MOOD) paradigm. Such a procedure enables to provide genuine gains in accuracy both for one- and two-dimensional problems involving non-smooth flows when compared to classical SPH methods.
\end{abstract}

Keywords: High-order methods; Smooth Particle Hydrodynamics; Meshless methods; Multi-dimensional Optimal Order Detection; Moving Least Squares

\section{Introduction}

The Smooth Particle Hydrodynamics (SPH) method is based on a Lagrangian formulation of the Navier-Stokes equations and is widely used in complex CFD applications. Initially introduced in the 1970s by Lucy [1], Gingold 
and Monaghan [2] for astrophysical applications, SPH is now applied to a wide variety of applications such as porous media flow, magnetohydrodynamics, penetration, shock damage, explosions, etc. We refer to [3,4] for a general review on the SPH applications.

Most SPH formulations rely on the artificial viscosity approach [1-3] to prevent the numerical approximation from oscillating near sharp fronts or discontinuities. The use of artificial viscosities, however, leads to a significant loss of accuracy in flows involving shocks or contact discontinuities: numerical diffusion leads to excessive smearing of shock fronts and strong glitches near contact discontinuities [5,6].

In SPH formulations, a particle represents a finite piece of volume with an associated mass, suggesting a link between the finite volume method and the SPH technique. Several authors couple a Riemann solver with the SPH framework and, more generally for meshless methods [5,7-12], where the interaction between two particles is handled by the Riemann problem solution at the midpoint distance. Such a close relationship has led to the question of whether finite volume methods do really require a mesh or not [13].

We aim to develop a new accurate, stable and low-dissipative SPH Riemann-based method. The technique presented here is based on the SPH-ALE formulation introduced by Vila, see [11,12]. In the SPH-ALE approach the dissipation required for stabilization is introduced by Riemann solvers instead of adding an explicit artificial viscosity term. In SPH-ALE, the Riemann problem is solved between two neighboring particles on the direction of the line connecting them. Each of these two particles defines the usual left of right states in Riemann solvers terminology. The solution from the Riemann solver introduces the necessary and sufficient dissipation to stabilize the scheme. High order is achieved by using reconstructions based on Taylor series expansions to provide an accurate numerical fluxes evaluation at the midpoint between two interacting particles. In [14] a SPH-ALE technique for incompressible flows has been presented using slope limiters for stabilization.

Here we present an SPH scheme that combines the basic framework proposed by Vila with the Multidimensional Optimal Order Detection (MOOD) paradigm [15-17]. The use of MOOD provides robust and accurate stability properties to the scheme. Unlike many other stabilization procedures where the limiting stage is performed a priori, that is, before the flux computation and solution update, the MOOD limiting procedure is performed a posteriori. Indeed, a candidate solution based on high-order approximated fluxes is computed and the numerical regularity is quantified to detect those cells where non-physical oscillations occur. A correction is carried out to locally reduce the order of the scheme and provide a more robust approximation.

High-accuracy is achieved through polynomial reconstruction of the variables evaluated at the integration points when solving the local Riemann problems. Moving Least Squares (MLS) approximations are used to compute the derivatives required in the polynomial reconstruction. The Moving Least Squares method [18,19] is a powerful technique for the approximation of functions from scattered data. It was first proposed by [20] in a finite volume framework for several sets of equations, including Euler and Navier-Stokes equations. In similar context, a secondorder SPH formulation using slope limiters or fifth-order WENO interpolation is presented in [21] for 1D problems. A 2D-WENO approach based on MLS has been developed by [22]. We also mention the work of [23,24] using a second-order scheme for the Magnetohydrodynamics equations derived as a Galerkin formulation that uses Riemann solvers and MLS.

The paper is organized as follows. In Section 2 we present the compressible Euler equations for gas dynamics, and we recall the SPH-ALE scheme of Vila and Ben Moussa. We briefly introduce the notations of the MLS reconstruction we shall use for increasing the accuracy of the scheme while a new MOOD paradigm in the SPH context is proposed in Section 3. We address, in Section 4, the numerical issue where we assess the accuracy and the robustness of the SPH-MOOD scheme. Finally, we draw some conclusions and perspectives in the last section.

\section{Discretization}

We consider the system of Euler equations, written in the following conservative form [11]

$$
L_{\boldsymbol{w}_{\text {frame }}} \boldsymbol{U}+\nabla \cdot\left(\mathcal{F}-\left(\boldsymbol{w}_{\text {frame }} \otimes \boldsymbol{U}\right)\right)=0
$$

with $L_{\boldsymbol{w}_{\text {frame }}} \boldsymbol{U}=\partial_{t} \boldsymbol{U}+\nabla \cdot\left(\boldsymbol{w}_{\text {frame }} \otimes \boldsymbol{U}\right)$ and $\boldsymbol{w}_{\text {frame }}$ stands for the velocity of the Lagrangian frame. Moreover, $\boldsymbol{U}$ and $\mathcal{F}=\left(\mathcal{F}_{x}, \mathcal{F}_{y}\right)$ are the vector of the conservative variables and the inviscid flux vector, namely 


$$
\boldsymbol{U}=\left(\begin{array}{c}
\rho \\
\rho u \\
\rho v \\
\rho E
\end{array}\right), \quad \mathcal{F}_{x}=\left(\begin{array}{c}
\rho u \\
\rho u^{2}+p \\
\rho u v \\
\rho u H
\end{array}\right), \quad \mathcal{F}_{y}=\left(\begin{array}{c}
\rho v \\
\rho u v \\
\rho v^{2}+p \\
\rho v H
\end{array}\right),
$$

where $\rho$ is the density, $\boldsymbol{u}=(u, v)^{T}$ the velocity, $E$ the specific total energy, $H=E+p / \rho$ and $p$ the pressure. We assume that the fluid is governed by an equation of state $p=P(\rho, \varepsilon)$ where $\varepsilon=E-\frac{1}{2}\left(u^{2}+v^{2}\right)$ represents the specific internal energy. In this work we have considered the ideal gas equation of state.

\subsection{Generic SPH formulation}

We use the SPH-ALE formulation proposed in [11,12]. The computational domain, $\Omega$, is discretized by a set of $N$ particles of effective volume $V_{i}$ with positions $\boldsymbol{r}_{i}=\left(x_{i}, y_{i}\right)^{T}$ for each particle $1 \leq i \leq N$. The discrete form of system (1)-(2) reads

$$
\begin{aligned}
& \frac{d\left(V_{i} \boldsymbol{U}_{i}\right)}{d t}=-\sum_{j=1}^{n_{i}} V_{i} V_{j} 2\left(\boldsymbol{G}_{i j}-\boldsymbol{H}_{i}\right) \cdot \nabla W_{i j}, \\
& \frac{d V_{i}}{d t}=\sum_{j=1}^{n_{i}} V_{i} V_{j} 2\left(\boldsymbol{w}_{i j}-\boldsymbol{w}_{i}\right) \cdot \nabla W_{i j},
\end{aligned}
$$

where Eq. (3) represents the evolution of the conservative variables and Eq. (4) describes the evolution of the effective volume associated to particle $i$. The evolution of particle position is given by a weighted average interpolation of the velocity [25]

$$
\frac{d \boldsymbol{r}_{i}}{d t}=\frac{\sum_{j=1}^{n_{i}} V_{j} \boldsymbol{w}_{j} W_{i j}}{\sum_{j=1}^{n_{i}} V_{j} W_{i j}} .
$$

In the previous equations, $\nabla W_{i j}=\nabla W\left(\boldsymbol{r}_{j}-\boldsymbol{r}_{i}, h_{i j}\right)$ is the gradient of the approximation kernel centered at particle $i$ and $n_{i}$ is the number of neighbor particles which are inside the stencil of the particle (see Fig. 1). The product $V_{i} V_{j} 2 \cdot \nabla W_{i j}$ corresponds to geometrical weights of the SPH-ALE approximation, since these terms depend only on the position of the particles $i$ and $j$ [26].

The term $\boldsymbol{H}=\boldsymbol{H}(\boldsymbol{U}, \boldsymbol{w})$ is the Lagrangian flux tensor of the Euler equations in a reference moving frame with velocity $\boldsymbol{w}$. Thus, $\boldsymbol{H}_{i}=\boldsymbol{H}\left(\boldsymbol{U}_{i}, \boldsymbol{w}_{i}\right)$ refers to $\boldsymbol{H}$ computed as a function of the state of the $i$ th particle:

$$
\boldsymbol{H}_{i}=\mathcal{F}\left(\boldsymbol{U}_{i}\right)-\boldsymbol{w}_{i} \otimes \boldsymbol{U}_{i} .
$$

The terms $\boldsymbol{w}_{i}, \boldsymbol{w}_{j}$ refer to the Lagrangian velocity of particles $i, j$. In an Eulerian frame $\boldsymbol{w}_{i}=\boldsymbol{w}_{j}=0$, and in a Lagrangian frame they are coincident with particle velocities $\boldsymbol{u}_{i}, \boldsymbol{u}_{j}$. The flux tensor defined in Eq. (6) leads to a flux difference formulation of the SPH scheme [22] and can be interpreted as a correction for the computation of the geometrical weights of the SPH-ALE approximation [26,27]. $\boldsymbol{G}_{i j}$ is the numerical flux tensor evaluated at the midpoint between particles $i$ and $j, \boldsymbol{r}_{i j}=\left(\boldsymbol{r}_{i}+\boldsymbol{r}_{j}\right) / 2$. It is computed using an exact or approximate Riemann solver in the co-moving frame. For example, the Rusanov flux [28] is defined as:

$$
\boldsymbol{G}_{i j}=\frac{1}{2}\left(\boldsymbol{H}_{i j}^{+}+\boldsymbol{H}_{i j}^{-}\right)-\frac{1}{2}\left|S_{i j}^{+}\right| \Delta \boldsymbol{U}_{i j} \cdot \boldsymbol{n}^{T},
$$

where $S_{i j}^{+}$is the maximum eigenvalue of the Jacobian matrix which writes in the Arbitrarian Lagrangian-Eulerian (ALE) framework

$$
S_{i j}^{+}=\max \left(\left(\boldsymbol{u}_{i j}^{+}-\boldsymbol{w}_{i j}\right) \cdot \boldsymbol{n}_{i j}+c_{i j}^{+},\left(\boldsymbol{u}_{i j}^{-}-\boldsymbol{w}_{i j}\right) \cdot \boldsymbol{n}_{i j}-c_{i j}^{-}\right),
$$




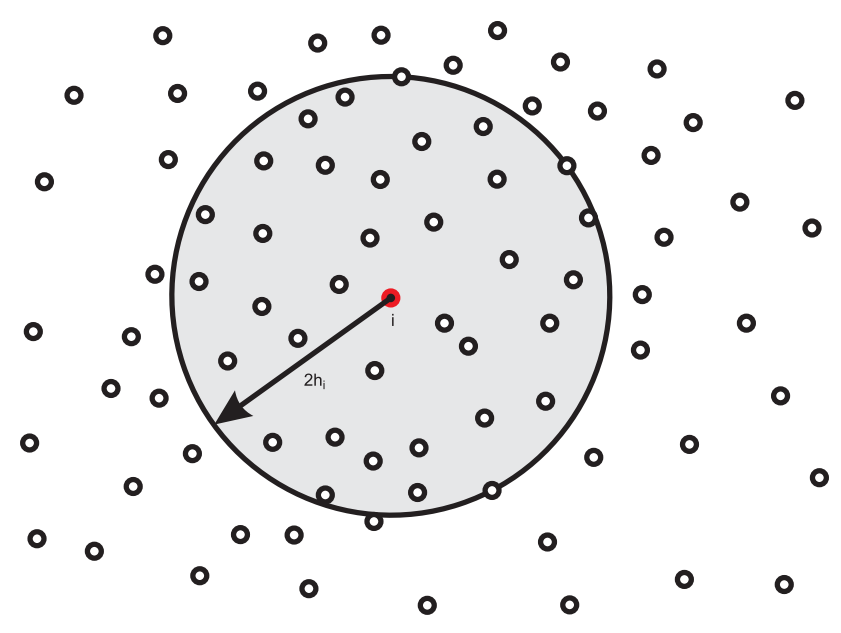

Fig. 1. Stencil of particle $i$ defined with the smoothing length $h_{i}$.

where $c_{i j}^{ \pm}$denotes the left and right sound speeds. Quantities $\boldsymbol{H}_{i j}^{-}=\boldsymbol{H}\left(\boldsymbol{U}_{i j}^{-}, \boldsymbol{w}_{i j}\right)$ and $\boldsymbol{H}_{i j}^{+}=\boldsymbol{H}\left(\boldsymbol{U}_{i j}^{+}, \boldsymbol{w}_{i j}\right)$ denote the flux approximations of $\boldsymbol{H}$ on the left and right sides of the integration point (midpoint between particles $i$ and $j$ ) $\boldsymbol{r}_{i j}$ with the positive orientation given by $\boldsymbol{r}_{j}-\boldsymbol{r}_{i}$ and represented by the normalized vector $\boldsymbol{n}_{i j}$ while $\Delta \boldsymbol{U}_{i j}=\boldsymbol{U}_{i j}^{+}-\boldsymbol{U}_{i j}^{-}$ is the jump of the reconstructed conservative vectors. Moreover, the term $\boldsymbol{w}_{i j}$ is the velocity of the reference frame. In an Eulerian frame, term $\boldsymbol{w}_{i j}=0$ whereas in a Lagrangian frame $\boldsymbol{w}_{i j}=\boldsymbol{u}_{i j}=\left(\boldsymbol{u}_{i j}^{+}+\boldsymbol{u}_{i j}^{-}\right) / 2$.

Note that in this formulation, Riemann solvers are used to introduce the amount of numerical dissipation required for stabilization, whereas in classical SPH approaches stabilization is based on the explicit introduction of numerical viscosity (see Appendix).

The kernel function $W_{i j}$ plays a crucial role in the SPH formulation. It is used to weight the different particles that contribute to the approximation. A wide variety of kernel functions are described in the literature [29-33] such as spline or exponential functions. We shall use the popular cubic spline kernel despite it not being the only or possibly the best option in terms of accuracy [34]:

$$
W_{i j}=\alpha_{d} \begin{cases}1-\frac{3}{2} q_{i j}^{2}+\frac{3}{4} q_{i j}^{3} & q_{i j} \leq 1, \\ \frac{1}{4}\left(2-q_{i j}\right)^{3} & 1<q_{i j} \leq 2, \\ 0 & q_{i j}>2,\end{cases}
$$

where $q_{i j}$ is defined as $q_{i j}=\left\|\boldsymbol{r}_{j}-\boldsymbol{r}_{i}\right\| / h_{i j}$. The term $h_{i j}$ represents the smoothing length. We adopt the variable smoothing length strategy for $h$ proposed by $[35,36]$

$$
h_{i j}=\frac{1}{2}\left(h_{i}+h_{j}\right), \quad \text { with } h_{i}=\sigma V_{i}^{\frac{1}{D}}
$$

where $D$ is the space dimensions number and $\sigma$ is a constant parameter. Note that the value of $\sigma$ determines the number of neighbors in the compact support of each kernel. Here we shall set to $\sigma=2$ in the numerical applications. The parameter $\alpha_{d}$ is a normalization constant so that the partition of the unity property holds. For one-dimensional geometries, we take $\alpha_{D}=1 / h_{i j}$ whereas $\alpha_{D}=10 /\left(7 \pi h_{i j}^{2}\right)$ for the two-dimensional case [29].

Remark. The main objective of this work is the extension of the MOOD paradigm to SPH methods, thus the choice of the formulation is not specific to the new limiting procedure we proposed and the MOOD technique suits well with any stable SPH formulation based on Riemann solvers.

\subsection{High-order reconstruction and Moving Least Squares approximations}

To improve the accuracy of the numerical method, we increase the order of the approximations of $\boldsymbol{U}_{i j}^{+}$and $\boldsymbol{U}_{i j}^{-}$that we plug into the numerical flux to compute $\boldsymbol{H}_{i j}^{+}$and $\boldsymbol{H}_{i j}^{-}$in Eq. (7). The quadratic reconstruction associated to particle 
$i$ and evaluated at $\boldsymbol{r}_{i j}$ reads

$$
\boldsymbol{U}_{i j}^{+}=\boldsymbol{U}_{i}+\nabla \boldsymbol{U}_{i} \cdot\left(\boldsymbol{r}_{i j}-\boldsymbol{r}_{i}\right)+\frac{1}{2}\left(\boldsymbol{r}_{i j}-\boldsymbol{r}_{i}\right)^{T} \nabla^{2} \boldsymbol{U}_{i}\left(\boldsymbol{r}_{i j}-\boldsymbol{r}_{i}\right),
$$

where the gradient $\boldsymbol{\nabla} \boldsymbol{U}_{i}$ and the Hessian matrix $\nabla^{2} \boldsymbol{U}_{i}$ involve the successive derivatives of $\boldsymbol{U}$. These terms are computed using MLS approximations.

Remark. The numerical scheme presents a second-order convergence at most. This is due to the discretization of the divergence of the convective terms in the Euler equation [37]. The approach presented in this work will allow us to improve the accuracy as we increase the order of the reconstruction, even though the global asymptotic order of the scheme will not exceed two.

We give a brief overview of the Moving Least Squares (MLS) technique for introducing the notations. We refer the reader to [18,20] for a complete description of the method. For a given set of $n_{i}$ values $\phi_{j}$ of position $\boldsymbol{r}_{j}$ in the neighbor of particle $i$ (see Fig. 1), the MLS approximation at point $\boldsymbol{r}=(x, y)^{T}$ is given by

$$
\widehat{\phi}(\boldsymbol{r})=\sum_{j=1}^{n_{i}} \Psi_{j}(\boldsymbol{r}) \phi_{j}
$$

where the associated shape functions, gathered in vector $\boldsymbol{\Psi}=\left(\Psi_{1}, \Psi_{2}, \ldots, \Psi_{n_{i}}\right) \in \mathbb{R}^{n_{i}}$, are computed by

$$
\boldsymbol{\Psi}^{T}(\boldsymbol{r})=\boldsymbol{p}^{T}(\boldsymbol{r}) \boldsymbol{M}^{-1}(\boldsymbol{r}) \boldsymbol{P}(\boldsymbol{r}) \boldsymbol{W}_{M L S}(\boldsymbol{r})
$$

where $\boldsymbol{p}^{T}(\boldsymbol{r})=\left(1, x, y, x^{2}, y^{2}, x y, \ldots\right) \in \mathbb{R}^{m}$ is the $m$-dimensional basis functions vector, $\boldsymbol{P}$ is a $m \times n_{i}$ matrix where the basis functions are evaluated at each point of the stencil, namely $P=\left[\boldsymbol{p}^{T}\left(\boldsymbol{r}_{j}\right)\right]_{i}$ and $\boldsymbol{M}(\boldsymbol{r})$ is the $m \times m$ moment matrix given by

$$
\boldsymbol{M}(\boldsymbol{r})=\boldsymbol{P}(\boldsymbol{r}) \boldsymbol{W}_{M L S}(\boldsymbol{r}) \boldsymbol{P}^{T}(\boldsymbol{r}) .
$$

Diagonal matrix $\boldsymbol{W}_{M L S}(\boldsymbol{r})$ is derived from the kernel function evaluated at $\boldsymbol{r}_{j}-\boldsymbol{r}_{i}$ for the $n_{i}$ neighboring particles. In practice, we take in the numerical applications the truncated exponential function [38]

$$
W_{i j}=W\left(\boldsymbol{r}_{j}, \boldsymbol{r}_{i}, s_{x}\right)=\frac{e^{-\left(\frac{d}{c}\right)^{2}}-e^{-\left(\frac{d_{m}}{c}\right)^{2}}}{1-e^{-\left(\frac{d_{m}}{c}\right)^{2}}}
$$

with $d=\left|\boldsymbol{r}_{j}-\boldsymbol{r}_{i}\right|, d_{m}=2 \max \left(\left|\boldsymbol{r}_{j}-\boldsymbol{r}_{i}\right|\right)$ and $d_{m}$ the smoothing length. The coefficient $c$ is defined by $c=\frac{d_{m}}{s_{x}}$ and $s_{x}=1$ is the shape parameter of the kernel. Note that even though it is possible to use the same kernel function for the computation of MLS shape functions and the SPH discretization of the Euler equations, we have experimented with two different kernels to provide more flexibility and future extensions for the MOOD paradigm.

High-order reconstruction (Eq. (11)) requires the computation of accurate derivatives, namely

$$
\nabla \widehat{\phi}=\sum_{j=1}^{n_{i}} \phi_{j} \nabla \Psi_{j}(\boldsymbol{r}) .
$$

We refer the interested reader to [20,38] for a detailed description of the computation of MLS derivatives.

\section{The Multi-dimensional Optimal Order Detection (MOOD) method}

It is well-known that high-order reconstructions lead to spurious numerical oscillations in the vicinity of discontinuities (the so-called Gibbs phenomenon). Limiting/stabilizing procedures such as artificial viscosity, MUSCL with slope limiter, or ENO/WENO are applied to locally increase the numerical diffusion for eliminating the nonphysical oscillations. All these methods are tagged a priori since the limiting procedure is performed with the data at time $t^{n}$ to prevent the approximation at time $t^{n+1}$ from oscillating. Recently, a new paradigm, based on an a posteriori 

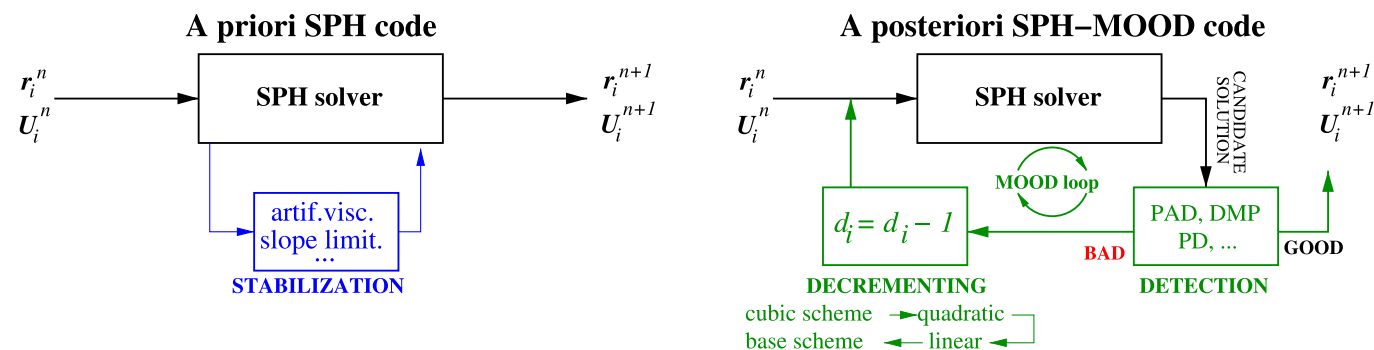

Fig. 2. Left: Sketch of a classical a priori stabilized SPH code. The stabilization is built in the solver through artificial viscosity, slope limiter, etc. Right: Sketch of an a posteriori SPH-MOOD like code. The MOOD loop assures that a BAD particle is detected when the candidate solution at time $t^{n+1}$ is not valid. For those particles only re-computation occurs with a less accurate numerical scheme after some decrementing. GOOD particles contrarily are accepted and are not recomputed.

limiting, has been proposed in [15,16] (the MOOD method). Here we extend the methodology to the context of SPH formulation.

The fundamental idea behind the MOOD paradigm is to determine, a posteriori, the optimal order of the polynomial reconstruction for each particle that provides the best compromise between accuracy and stability. To this end, a candidate solution, $\boldsymbol{U}^{\star}$, is evaluated as a potential approximation for time $t^{n+1}$. Then the candidate solution is confronted with a series of tests, named detectors, to check whether the approximation is acceptable or not according to the some predefined smoothness criteria. For the troubled/bad cells, i.e. where the solution is declared as not valid, the candidate solution is discarded and recomputed, starting again from data at $t^{n}$ but with a more dissipative scheme that uses a lower polynomial degree for the reconstruction.

\subsection{MOOD loop}

More precisely, the MOOD method is based on two main ingredients: a Particle Polynomial Degree (PPD), and a chain of detectors to assess the validity of the candidate solution. The PPD indicates the degree of the polynomial function we shall use to compute the candidate solution $\boldsymbol{U}^{\star}$. The flux is evaluated at the midpoint $\boldsymbol{r}_{i j}$ between particles $i$ and $j$ taking the polynomial representation of degree $\min (P P D(i), P P D(j))$. The chain detector controls the admissibility of the resulting solution and the particles PPD is decremented where a detector is activated. The MOOD loop then consists in iterating through the PPD map, initialized with a maximal order $\left(d_{\max }=3\right)$, and in decreasing the degree of defected particle pointed by the chain detector. If the PPD map is modified, the candidate solution is discarded and recomputed with the new PPD map else the candidate solution turns to be the approximation at $t^{n+1}$. It is of crucial importance to note that only the altered cells have to be computed again, which saves a significant amount of computational resources. In the worst situation, i.e. the PPD map has all its entries equal to zero, we obtain the so-called parachute base scheme, using the piecewise constant approximation, and providing, de facto, the first-order and robust Vila scheme. The solution computed with this approximation is assumed to be always valid. In Fig. 2, left panel, we sketch the classical a priori stabilized SPH code through artificial viscosity or slope limiter, while we display in the right panel the a posteriori SPH-MOOD procedure.

Remark. It is proven that the MOOD loop always terminates [15], either with all particles being updated by the parachute scheme (i.e. all PPD equal to 0), or if all particles being valid according to the chain of detection criteria.

\subsection{Chain detectors}

In order to achieve stability within our SPH formulation, a chain of detectors is built, comprising three elementary detectors, namely the Physical Admissibility Detection (PAD), the Discrete Maximum Principle detector (DMP) and the Plateau Detector (PD), assembled together in the so-called chain detector given in Fig. 3. Notice that the order of the elementary detectors is important since the activation of one detector determines the use of another detector. We detail in the next paragraph the definition and the implementations of the three detectors. 


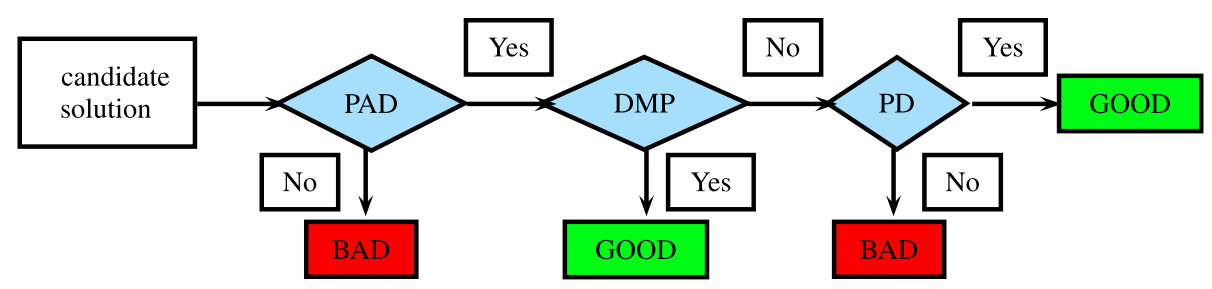

Fig. 3. Chain detector composed of successive elementary detectors.

\subsubsection{Physical Admissibility Detection (PAD)}

The Physical Admissibility Detection (PAD) requires that the candidate solution remains physically admissible. This set of criteria is intrinsically dependent on the set of PDEs which are being solved. In this work we solve the Euler system of conservation laws, and therefore density and pressure must remain non-negative. For SPH formulations we consider that a candidate solution is physically admissible if it satisfies the following conditions:

- Positivity of the density: $\rho_{i}^{\star}>0$,

- Positivity of the pressure: $p_{i}^{\star}>0$ (or equivalently $\varepsilon_{i}^{\star}>0$ ),

- Positivity of the cell volume: $V_{i}^{\star}>0$.

Note that those conditions do not prevent the solution from oscillating, but rather guarantee that the code will not crash (non-negative sound velocity) due to unphysical state variables.

\subsubsection{Discrete Maximum Principle (DMP)}

The Discrete Maximum Principle (DMP) criterion prevents spurious numerical oscillations from appearing in the vicinity of discontinuities and sharp fronts. An admissible candidate solution fulfills the DMP if it satisfies

$$
\min _{j \in \mathcal{V}_{i}}\left(\rho_{j}^{n}, \rho_{i}^{n}\right) \leq \rho_{i}^{\star} \leq \max _{j \in \mathcal{V}_{i}}\left(\rho_{j}^{n}, \rho_{i}^{n}\right)
$$

where $\mathcal{V}_{i}$ is a set of neighbor particles. If a particle does not fulfill the criterion, it follows that an extremum is detected and a potential oscillation may happen. Such a situation is considered as problematic, and therefore the particle should be recomputed with a more dissipative scheme.

The DMP criterion is very robust, but it may lead to an overly dissipative scheme. Indeed, a new local extremum is not per se the starting point of a spurious oscillation, but may rather correspond to a physically relevant event (a compression for example). Nonetheless, all spurious oscillations do emerge from a new local extremum. Additional detectors have been designed in [15-17] to differentiate physically relevant extremum from spurious oscillations, in order to achieve numerical schemes with very high-order of accuracy. However, due to the effective second-order of the SPH scheme, such detectors are not required here.

In the application of MOOD to finite volume formulations, $\mathcal{V}_{i}$ is constituted of the cells sharing a face (in 3D) or an edge (in 2D) with the control volume $i$ [15]. In SPH formulations, we define $\mathcal{V}_{i}$ as the set of closest particles coming from different directions. To this end, we split the reconstruction stencil of the particle $i$ into four sub-domains, as displayed in Fig. 4. For each sub-domain, we choose the closest particle to particle $i$ and include it in $\mathcal{V}_{i}$. Since particles are moving, one should check if the candidate position of the particle $i$ leaves $\mathcal{V}_{i}$ (see Fig. 4). If that is the case, the DMP detector defined by relation (17) is no longer valid, and the PPD for particle $i$ is set to zero. Another scenario has been also tested where we use smaller time steps until this condition is fulfilled.

\subsubsection{Plateau Detector (PD)}

Assume that a smooth solution is constant on a sub-domain $D$. Due to the truncation for real numbers representation on computer, the constant solution is numerically contaminated by a noise which provides artificial extrema of relative order $10^{-15}$. The DMP detector incorrectly interprets the noise as local extrema, leading to a dramatic order reduction while the solution is mainly constant. To overcome such a drawback, we introduce the Plateau Detector, which releases the DMP criterion when the solution is almost constant.

For a particle $i$ of stencil $\mathcal{V}_{i}$, the Plateau Detector is activated if

$$
\max _{j \in \mathcal{V}_{i}}\left(\left|\rho_{i}^{\star}-\rho_{j}^{\star}\right|\right) \leq \varepsilon_{P}
$$




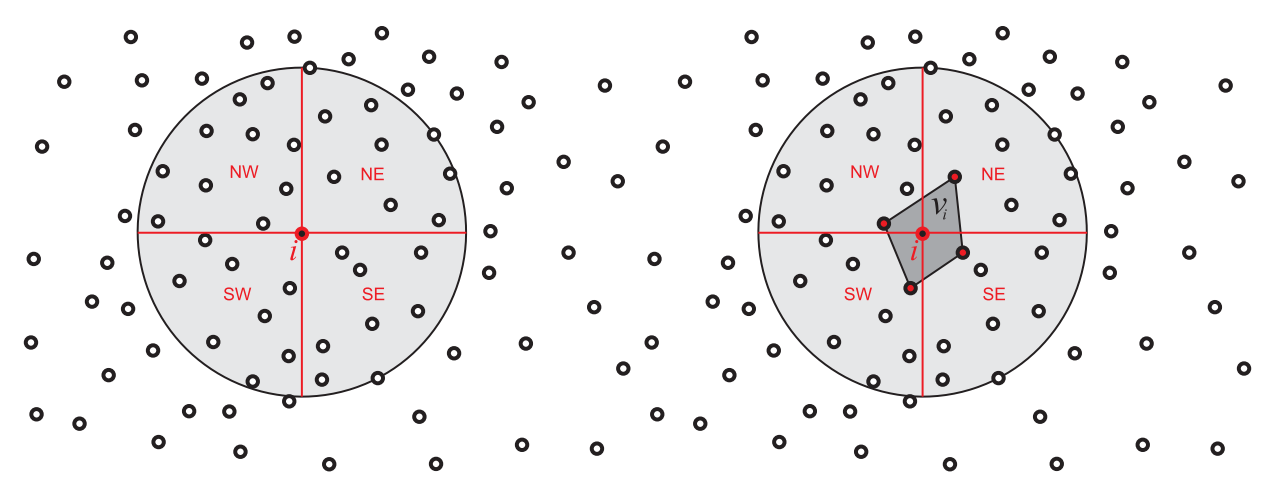

Fig. 4. Sketch of the stencil of particle $i$ divided into four sub domains needed for the DMP (NW, NE, SE and SW).

where $\varepsilon_{P}>0$ is the Plateau Detector threshold. In this work we have set $\varepsilon_{P}=10^{-15}$. If the DMP is activated for the particle $i$, we check the plateau criterion. If the PD is activated, then we release the DMP detector maintaining the maximum order.

\section{Numerical tests and examples}

We present representative numerical simulations to assess the quality of the SPH-MOOD scheme to produce accurate and robust approximations, and demonstrate the suitability of the method for solving the Euler equations in multidimensional form. All the numerical examples have been computed using a third-order Runge-Kutta scheme for time integration [39]. The initial particles volumes, $V_{i}$, are determined such that

$$
\int_{\Omega_{i}} W d \Omega_{i}=\sum_{j}^{n_{i}} V_{j} W_{i j}
$$

following [24,34]. Several SPH-MOOD versions have been implemented using different Taylor reconstructions and will be compared to the first-order Vila scheme [11], which is henceforth referred to as the SPH parachute scheme.

In order to assess the performance of the SPH-MOOD scheme, several benchmarks have been carried out that we list hereafter.

$2 D$ pure reconstruction test. This two-dimensional test case provides an assessment of the proposed reconstruction procedure.

$2 D$ Ringleb's flow problem. The two-dimensional problem provides a smooth solution we use to measure the effective order of accuracy of the tested SPH scheme.

$1 D$ Sod like shock tubes. Two one-dimensional tests presenting simple non-interacting waves (rarefaction, contact discontinuity and shock) are carried out to assess the robustness of the schemes. Also we use this test to prove that improvement can be gained if a less dissipative Riemann solver is employed in replacement of the Rusanov one.

$2 D$ circular blast waves. We have performed the simulations for two situations where three cylindrical waves are generated. The tests check the ability of the schemes to preserve the axisymmetric symmetry but also to evaluate the accuracy gain with the MOOD procedure faced to the traditional methods.

\section{1. $2 D$ pure reconstruction test}

We begin by studying the accuracy of the reconstruction procedure on $2 \mathrm{D}$ square domain $\Omega=[-1.5,1.5] \times$ $[-1.5,1.5]$ without considering any fluid flow. The assessment of the accuracy is performed by setting the point value of a known function to each particle. In this example we define the function to be reconstructed as

$$
f(\boldsymbol{r})=a e^{-\frac{|r|^{2}}{2 b}}
$$

where $\boldsymbol{r}$ is the position of the particle, $a=1 /(\sqrt{2 b \pi})$ and $b=0.20$. 

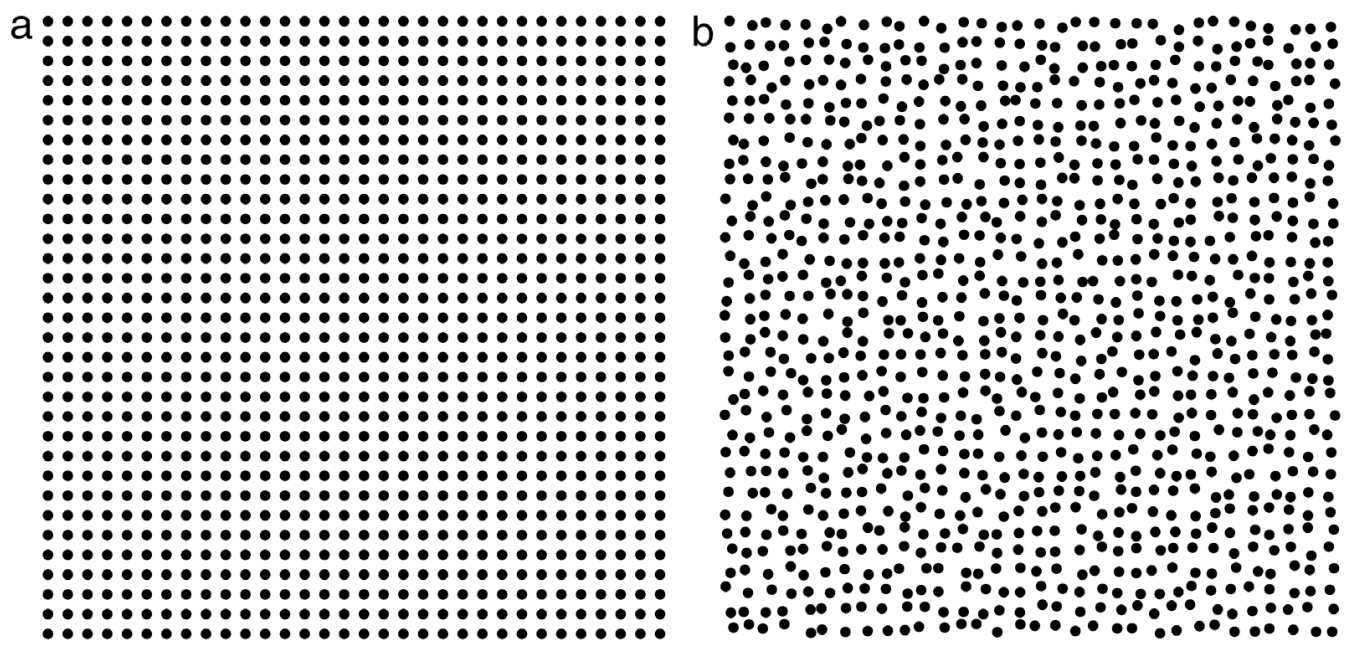

Fig. 5. Particle distributions used for 2D Reconstruction test case. Equidistant (a) and random (b) distributions of 1024 particles.

MLS derivatives are computed at the position of each particle and Taylor reconstruction is performed in order to compute the value at the midpoint between particles. Note that in the SPH computations, the order of the Taylor reconstruction is controlled by the MOOD procedure.

We have performed this test case using both, an equidistant and a random particle distribution, as shown in Fig. 5. The random set is obtained by perturbing randomly the equidistant distribution.

$L_{2}^{N}$ and $L_{\infty}^{, N}$ norms of the reconstruction error are computed for each distribution with $N$ particles. Convergence rates are obtained by using the errors obtained for two different sets with $N_{1}$ and $N_{2}$ particles by using the formula

$$
O^{N}=\frac{\log \left(L^{N_{1}} / L^{N_{2}}\right)}{\log \left(\sqrt{N_{2} / N_{1}}\right)} .
$$

Errors and convergence rates for quadratic and cubic reconstructions are reported in Tables 1 and 2 for equidistant and random distributions of particles. It is observed that the optimal orders of accuracy are recovered independently of the location of the particles.

\subsection{Ringleb's flow}

In this section we study the rate of convergence for the SPH-MOOD-MLS method for an inviscid compressible fluid. The flow solution is obtained as a solution of the hodograph equation [40]. The computational domain $\Omega=[-1.15,-0.75] \times[0.15,0.55]$ is discretized using several different sets of particle distributions. Each distribution has about two times more particles than the previous one. For this test case, the particle motion is Eulerian. In order to check the formal order of accuracy of our numerical solver, we compute the $L_{2}$ norm of the entropy error as

$$
L_{2}^{e n t, N}=\sqrt{\frac{1}{\Omega} \int_{\Omega}\left(\frac{p_{N} / \rho_{N}^{\gamma}-p_{\infty} / \rho_{\infty}^{\gamma}}{p_{\infty} / \rho_{\infty}^{\gamma}}\right)^{2} d \Omega}
$$

where $\gamma=7 / 5$, and $\rho_{N}, p_{N}$ is the numerical solution, computed with $N$ particles at final time, and $\rho_{\infty}, p_{\infty}$ stand for the density and pressure of the exact solution respectively. For two sets of particles with respective number $N_{1}$ and $N_{2}$, we expect the global entropy error to decrease, and, to reach an asymptotic numerical order of convergence computed as

$$
O_{2}^{e n t, N}=\frac{\log \left(L_{2}^{e n t, N_{1}} / L_{2}^{e n t, N_{2}}\right)}{\log \left(\sqrt{N_{2} / N_{1}}\right)} .
$$


Table 1

Accuracy orders $O_{2}^{N}, O_{\infty}^{N}$ and $L_{2}^{N}, L_{\infty}^{N}$ norms of the reconstruction error for the 2D reconstruction test cases using an equidistant distribution of particles.

\begin{tabular}{lrllll}
\hline & \multicolumn{1}{c}{$N$} & $L_{2}^{N}$ error & $O_{2}^{N}$ & $L_{\infty}^{N}$ error & $O_{\infty}^{N}$ \\
\hline \multirow{3}{*}{ Quadratic reconstruction } & 1024 & $1.41 \times 10^{-4}$ & - & $6.92 \times 10^{-4}$ & - \\
& 2304 & $2.87 \times 10^{-5}$ & 3.923 & $1.35 \times 10^{-4}$ & 4.039 \\
& 4096 & $1.02 \times 10^{-5}$ & 3.612 & $4.53 \times 10^{-5}$ & 3.784 \\
& 9216 & $2.61 \times 10^{-6}$ & 3.356 & $1.10 \times 10^{-5}$ & 3.484 \\
& 16384 & $1.04 \times 10^{-6}$ & 3.189 & $4.29 \times 10^{-6}$ & 3.281 \\
Cubic reconstruction & 36864 & $2.88 \times 10^{-7}$ & 3.172 & $1.25 \times 10^{-6}$ & 3.051 \\
& 1024 & $1.56 \times 10^{-4}$ & - & $7.80 \times 10^{-4}$ & - \\
& 2304 & $2.91 \times 10^{-5}$ & 4.141 & $1.48 \times 10^{-4}$ & 4.105 \\
& 4096 & $8.96 \times 10^{-6}$ & 4.093 & $4.59 \times 10^{-5}$ & 4.064 \\
& 9216 & $1.73 \times 10^{-6}$ & 4.055 & $8.91 \times 10^{-6}$ & 4.043 \\
& 16384 & $5.43 \times 10^{-7}$ & 4.030 & $2.80 \times 10^{-6}$ & 4.024 \\
& 36864 & $1.07 \times 10^{-7}$ & 4.017 & $5.50 \times 10^{-7}$ & 4.013 \\
\hline
\end{tabular}

Table 2

Accuracy orders $O_{2}^{N}, O_{\infty}^{N}$ and $L_{2}^{N}, L_{\infty}^{N}$ norms of the reconstruction error for the $2 \mathrm{D}$ reconstruction test cases using a random distribution of particles.

\begin{tabular}{|c|c|c|c|c|c|}
\hline & $N$ & $L_{2}^{N}$ error & $O_{2}^{N}$ & $L_{\infty}^{N}$ error & $O_{\infty}^{N}$ \\
\hline \multirow{6}{*}{ Quadratic reconstruction } & 1024 & $2.09 \times 10^{-4}$ & - & $1.59 \times 10^{-3}$ & - \\
\hline & 2304 & $4.38 \times 10^{-5}$ & 3.849 & $3.37 \times 10^{-4}$ & 3.824 \\
\hline & 4096 & $1.52 \times 10^{-5}$ & 3.673 & $1.25 \times 10^{-4}$ & 3.445 \\
\hline & 9216 & $3.76 \times 10^{-6}$ & 3.453 & $3.43 \times 10^{-5}$ & 3.186 \\
\hline & 16384 & $1.47 \times 10^{-6}$ & 3.253 & $1.29 \times 10^{-5}$ & 3.395 \\
\hline & 36864 & $4.12 \times 10^{-7}$ & 3.145 & $4.04 \times 10^{-6}$ & 2.865 \\
\hline \multirow{6}{*}{ Cubic reconstruction } & 1024 & $2.26 \times 10^{-4}$ & - & $1.59 \times 10^{-3}$ & - \\
\hline & 2304 & $4.34 \times 10^{-5}$ & 4.066 & $3.58 \times 10^{-4}$ & 3.681 \\
\hline & 4096 & $1.36 \times 10^{-5}$ & 4.031 & $1.14 \times 10^{-4}$ & 3.988 \\
\hline & 9216 & $2.63 \times 10^{-6}$ & 4.058 & $2.76 \times 10^{-5}$ & 3.493 \\
\hline & 16384 & $8.29 \times 10^{-7}$ & 4.007 & $7.96 \times 10^{-6}$ & 4.319 \\
\hline & 36864 & $1.64 \times 10^{-7}$ & 3.999 & $1.73 \times 10^{-6}$ & 3.760 \\
\hline
\end{tabular}

Errors and their associated convergence rates are reported in Table 3, while Fig. 6 displays the convergence curves as a function of $N$ in log-scale. We observe that the accuracy increases as the reconstruction is improved. However, the global order of the scheme remains a second-order one asymptotically. Nonetheless, gain in accuracy with respect to the base scheme using the proposed method is up to 6 orders of magnitude. The MOOD approach systematically improves the error as the degree of the Taylor approximation increases.

\subsection{ID Riemann problems}

Two one-dimensional test problems involving discontinuous solutions are considered to assess the robustness of the scheme. The solutions of the Riemann problems we consider are constituted of simple waves such as genuinely non-linear shock, contact discontinuity or rarefaction fan.

\subsubsection{Sod tube}

The first case (T1) is the Sod shock tube on the domain is $[0,1]$ characterized by the initial conditions

$$
\text { (T1) }(\rho, \mathrm{u}, \mathrm{p})= \begin{cases}(1,0,1), & \text { if } x \leq 0.5 \\ (0.125,0,0.1), & \text { otherwise. }\end{cases}
$$


Table 3

Accuracy orders $O_{2}^{\text {ent }, N}$ and $L_{2}^{\text {ent, } N}$ norm of entropy error for the Ringleb flow test case, using the SPH Base scheme and the proposed SPH-MOOD-MLS schemes with linear, quadratic and cubic Taylor reconstructions respectively.

\begin{tabular}{|c|c|c|c|c|c|c|c|}
\hline & $N$ & $L_{2}^{e n t, N}$ error & $O_{2}^{e n t, N}$ & & $N$ & $L_{2}^{e n t, N}$ error & $O_{2}^{e n t, N}$ \\
\hline \multirow{7}{*}{ Base scheme } & 1024 & $8.28 \times 10^{-4}$ & - & \multirow{7}{*}{ Linear reconstruction } & 1024 & $8.54 \times 10^{-7}$ & - \\
\hline & 2304 & $5.76 \times 10^{-4}$ & 0.898 & & 2304 & $3.84 \times 10^{-7}$ & 1.971 \\
\hline & 4096 & $4.42 \times 10^{-4}$ & 0.918 & & 4096 & $2.15 \times 10^{-7}$ & 2.012 \\
\hline & 9216 & $3.03 \times 10^{-4}$ & 0.933 & & 9216 & $9.60 \times 10^{-8}$ & 1.992 \\
\hline & 16384 & $2.31 \times 10^{-4}$ & 0.946 & & 16384 & $5.40 \times 10^{-8}$ & 1.998 \\
\hline & 36864 & $1.56 \times 10^{-4}$ & 0.957 & & 36864 & $2.40 \times 10^{-8}$ & 1.998 \\
\hline & 65536 & $1.18 \times 10^{-4}$ & 0.966 & & 65536 & $1.35 \times 10^{-8}$ & 2.007 \\
\hline \multirow{7}{*}{ Quadratic reconstruction } & 1024 & $3.69 \times 10^{-8}$ & - & \multirow{7}{*}{ Cubic reconstruction } & 1024 & $1.69 \times 10^{-8}$ & - \\
\hline & 2304 & $1.01 \times 10^{-8}$ & 3.202 & & 2304 & $4.13 \times 10^{-9}$ & 3.466 \\
\hline & 4096 & $3.30 \times 10^{-9}$ & 3.876 & & 4096 & $1.13 \times 10^{-9}$ & 4.499 \\
\hline & 9216 & $1.53 \times 10^{-9}$ & 1.892 & & 9216 & $9.54 \times 10^{-10}$ & 0.425 \\
\hline & 16384 & $4.90 \times 10^{-10}$ & 3.968 & & 16384 & $2.9 \times 10^{-10}$ & 4.138 \\
\hline & 36864 & $1.86 \times 10^{-10}$ & 2.384 & & 36864 & $1.38 \times 10^{-10}$ & 1.836 \\
\hline & 65536 & $9.67 \times 10^{-11}$ & 2.281 & & 65536 & $7.91 \times 10^{-11}$ & 1.930 \\
\hline
\end{tabular}

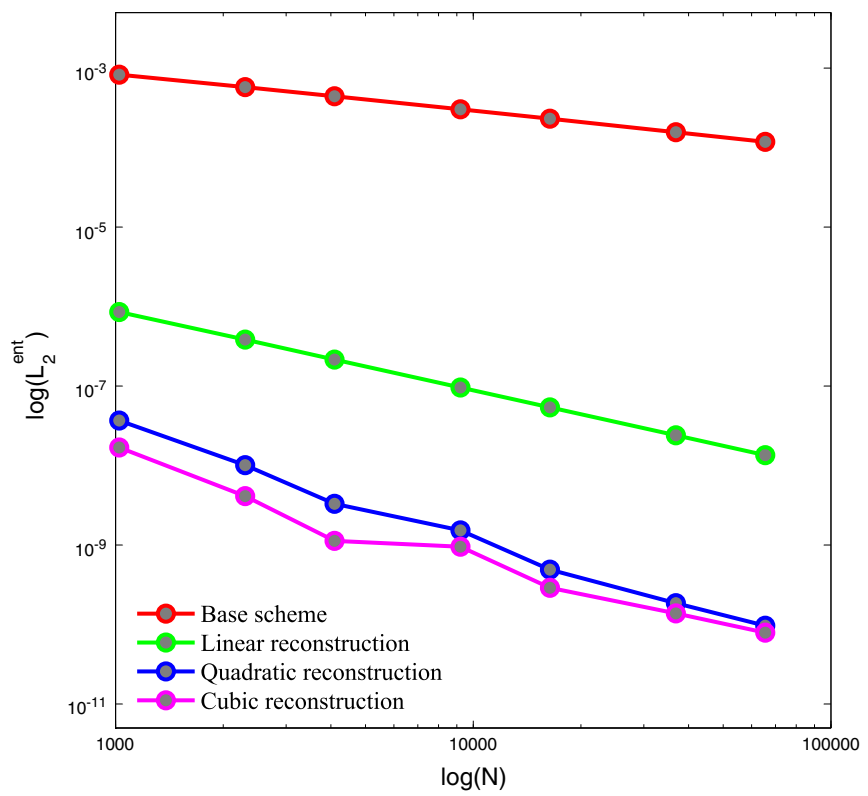

Fig. 6. Entropy errors $L_{2}^{\text {ent }, N}$ as a function of $N$ in log-scale for all SPH-MOOD schemes and the base scheme. Source: Data are taken from Table 3.

We use a discretization of 200 particles and solutions are advanced up to $t=0.2$. The exact solution comprises a left-moving rarefaction wave, a right-moving contact discontinuity, and a shock wave. We use a Taylor reconstruction until the third derivative controlled by the MOOD procedure, with Rusanov numerical flux.

Fig. 7 displays the numerical approximation compared with the exact solution. We note the very good agreement with the exact solution computed using the NUMERICA library, detailed in [41]. We also notice the absence of spurious oscillations. Moreover, we observe the great improvement in accuracy compared with the first-order scheme (the Vila's one).

Fig. 8 shows the results obtained for different number of particles. We observe that the approximations converge to the exact solution, and the curves for different variables obtained with 100 particles with the SPH-MOOD-MLS scheme are closer to the exact solution than those obtained with the base SPH scheme using 200 particles. Finally we present in Fig. 9 a comparison of our SPH scheme when the Rusanov flux (7) or the classical HLLC flux, 

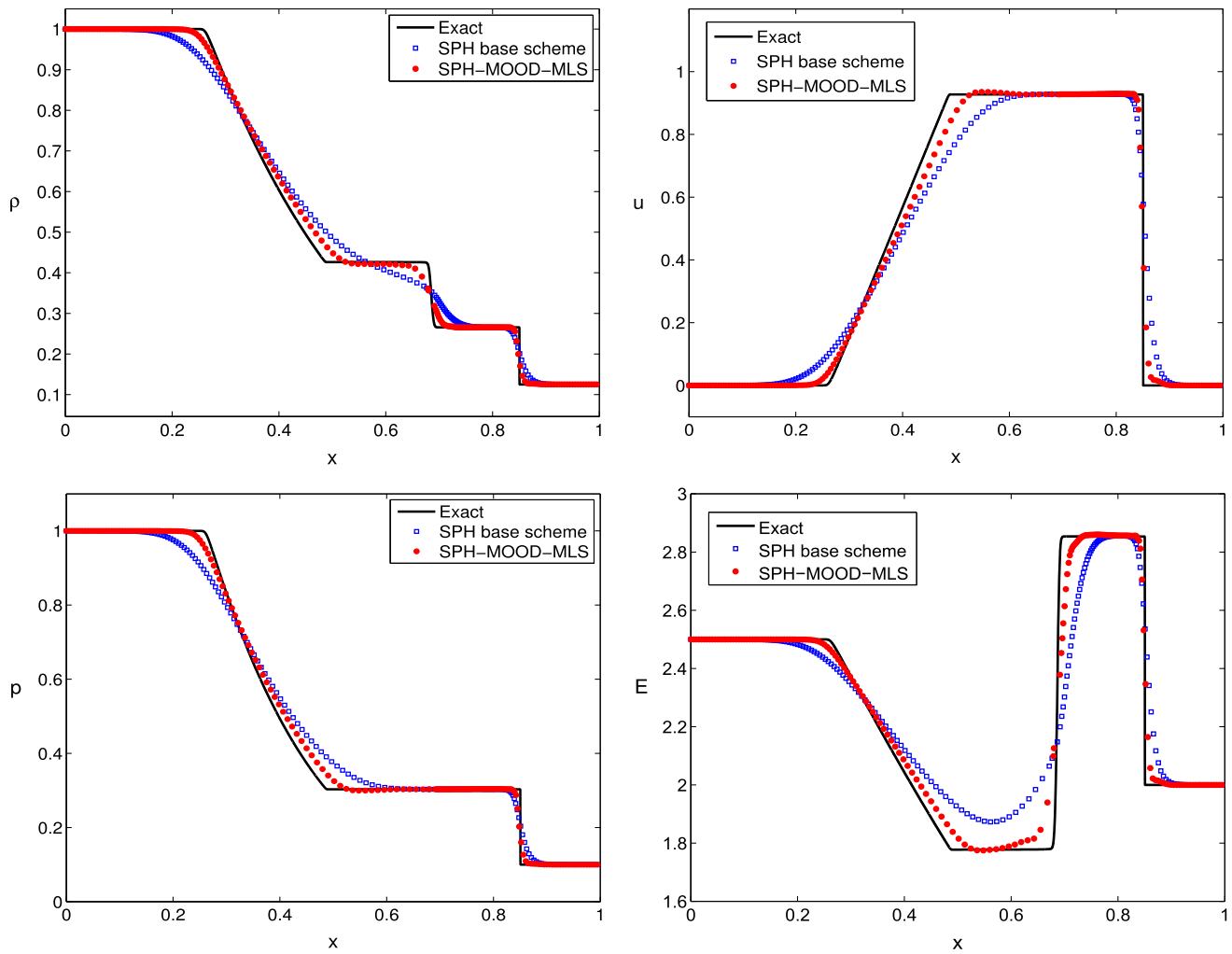

Fig. 7. Simulations results for the 1D Sod shock tube problem (T1) using 200 particles in the domain [0, 1]. We plot density (top-left), velocity (top-right), pressure (bottom-left), specific total energy (bottom-right). Results obtained using the SPH-MOOD-MLS method with Rusanov flux and a fourth-order Taylor reconstruction (filled circles), and using the SPH base scheme (empty squares).

see [41,42], are employed. While the accuracy of the solution remains essentially unchanged on the shock and rarefaction, it is truly improved on the contact discontinuity when the HLLC Riemann solver is employed. Note that other solvers could also be tested (Roe, Osher).

For the second test case (T2) detailed in [29,43], we consider a domain $\Omega=[-0.6,0.6]$ and the initial conditions are given by

$$
\text { (T2) }(\rho, \mathrm{u}, \mathrm{p})= \begin{cases}(1,0,1), & \text { if } x \leq 0.5 \\ (0.25,0,0.1795), & \text { otherwise. }\end{cases}
$$

A discretization using 400 particles is applied where the particles are distributed in two zones. In the first one (covering the domain $[-0.6,0]$ ) we put 320 equally distributed particles while in the second one (domain $[0,0.6]$ ) we place 80 particles. Computation is carried out until the final time, which is $t=0.2$.

Fig. 10 shows the density, velocity, pressure and internal energy curves at the final time. We also plot the numerical results obtained using an SPH method with artificial viscosity (AV) [29] which exhibits a better accuracy than the original first-order base scheme (Vila's SPH-ALE scheme). Unfortunately for the SPH with AV, we observe wiggles and kinks appearing in the pressure, velocity and energy plots. Conversely, Vila's scheme (base scheme), does not present these problems but, as we have already seen on the (T1) problem, it provides a solution with significant dissipation. We remark that the SPH-MOOD-MLS scheme proposed here obtains more accurate results than the artificial viscosity SPH method without numerical artifacts. We also observe the wiggles and kinks are appearing in the pressure, velocity and energy plots when using this scheme. The Vila scheme (base scheme), does not present these problems, but it gives a very dissipative solution. Finally, the SPH-MOOD-MLS scheme provides a more accurate approximation than the artificial viscosity SPH method without numerical artifacts. 

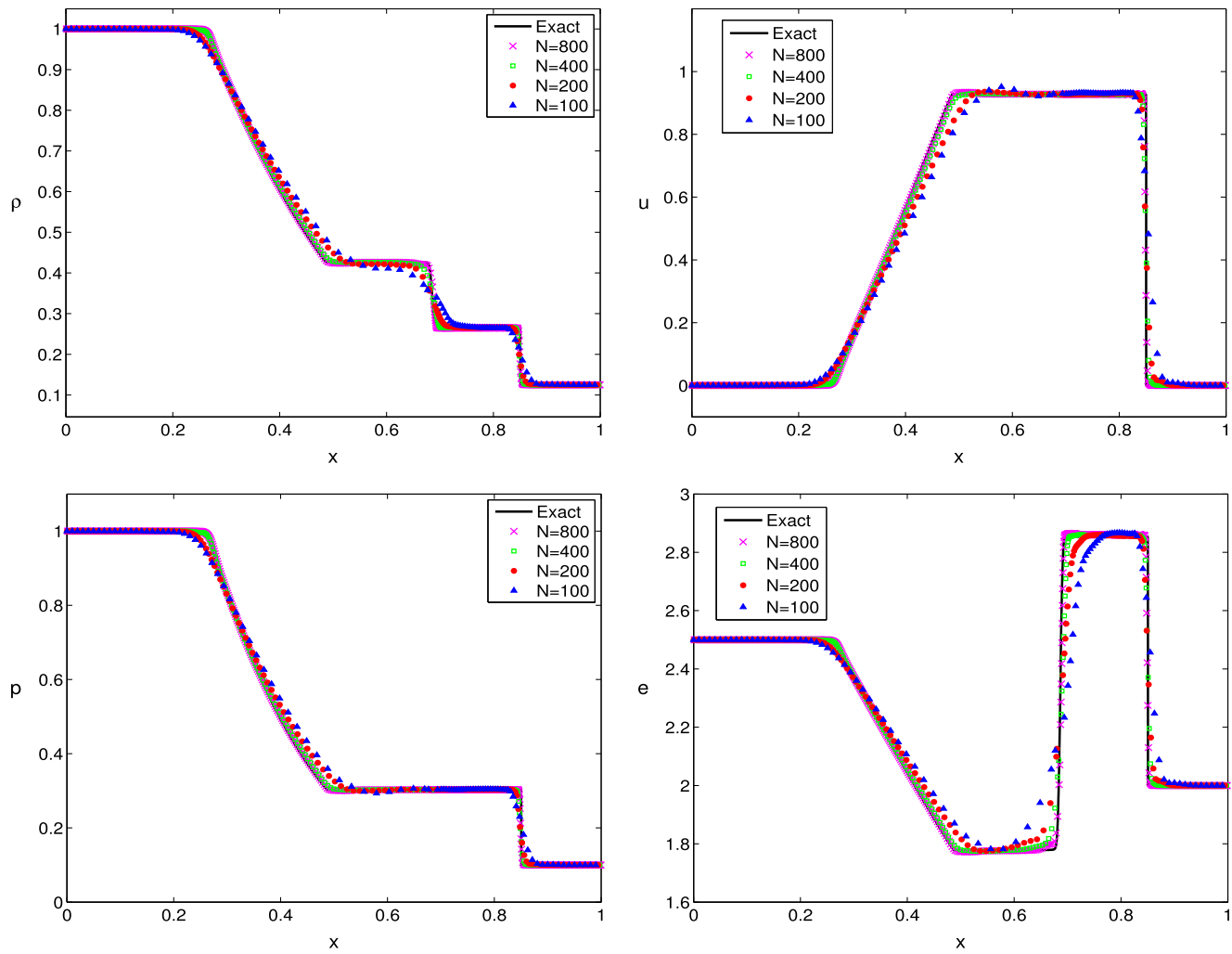

Fig. 8. Results for the 1D Sod shock tube problem (T1) using different number of particles in the domain [0, 1]. We plot density (top-left), velocity (top-right), pressure (bottom-left), specific total energy (bottom-right). Results obtained using the SPH-MOOD-MLS method with Rusanov flux and a fourth-order Taylor reconstruction.
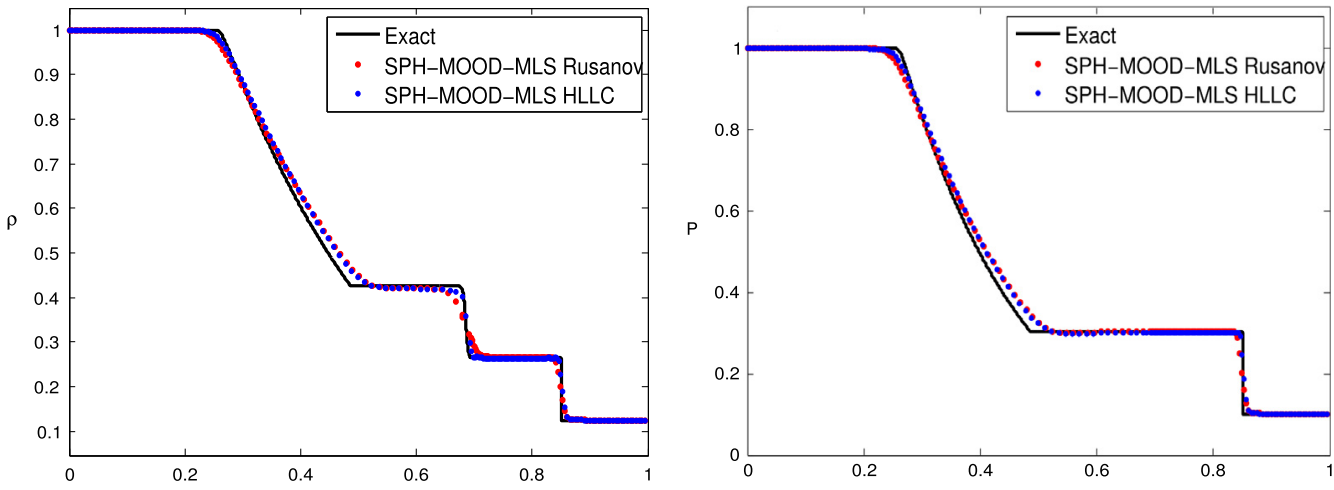

Fig. 9. Results for the 1D Sod shock tube problem (T1) in the domain $[0,1]$. We plot density (left) and pressure (right). Results obtained using the SPH-MOOD-MLS method with Rusanov flux (red) and HLLC flux (blue) and a fourth-order Taylor reconstruction. (For interpretation of the references to color in this figure legend, the reader is referred to the web version of this article.)

\subsection{Circular blast wave problems}

The computational domain for two 2D blast wave problems is an open disk with center in $(0,0)$ and radius $R=1.5$. In order to create the initial distribution of the particles, shown in Fig. 11, we first define the Delaunay triangulation of given sets of generators, then the particles are matched to the barycenters of the triangulation. A regular and radially symmetric and an unstructured set of 74651 generators are considered. As mentioned these generators are used as 

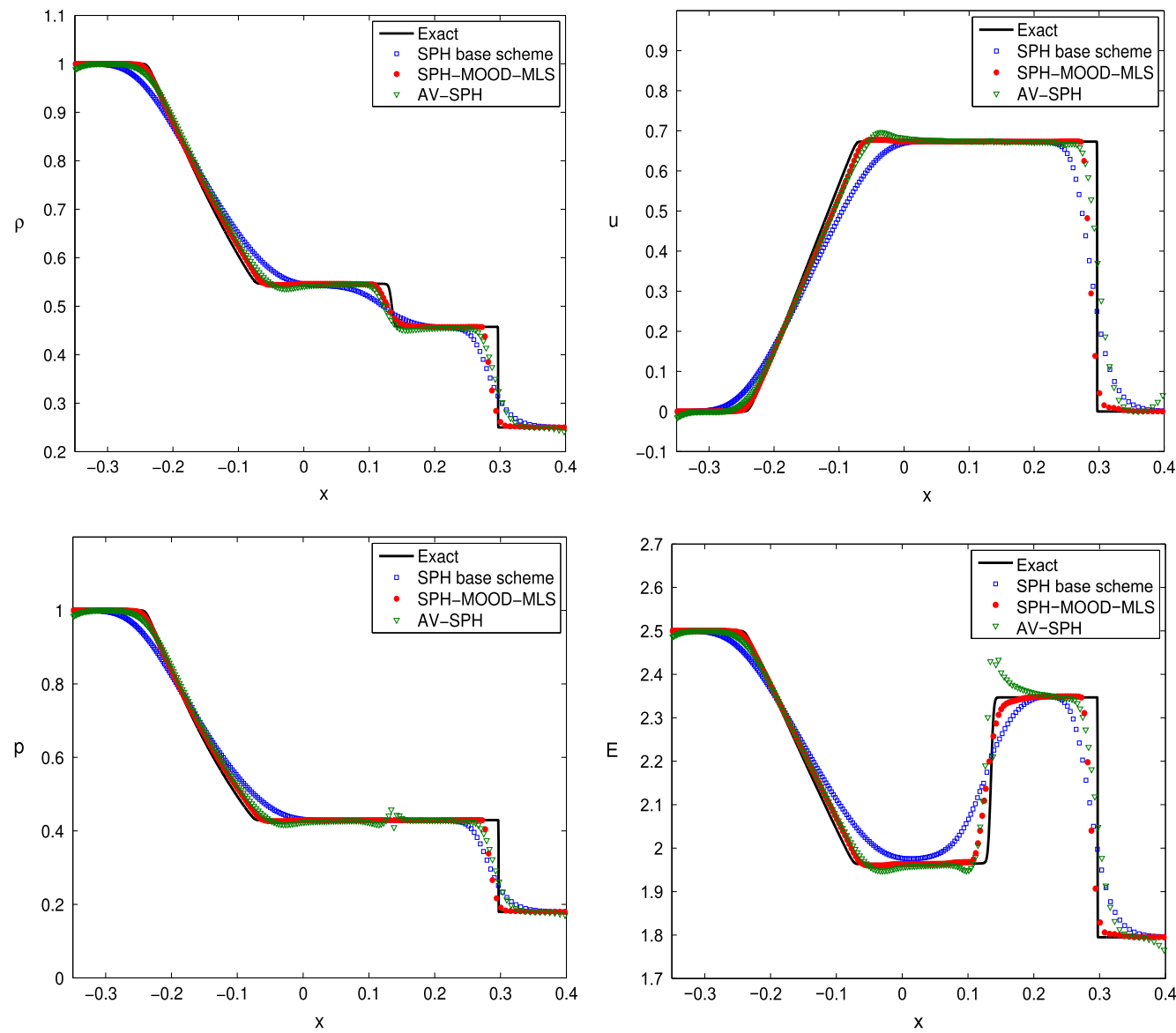

Fig. 10. Results for the 1D Sod shock tube problem (T2) using 400 particles in the domain $[-0.6,0.6]$, distributed as explained in the text. We plot density (top-left), velocity (top-right), pressure (bottom-left), specific total energy (bottom-right). Results obtained using the SPH-MOOD-MLS method with Rusanov flux and a fourth-order Taylor reconstruction (filled circles), and using the SPH base scheme (empty squares). We also plot the results obtained with an SPH method using artificial viscosity (AV-SPH) [29].

the initial vertex of the triangles of the associated and unique Delaunay triangulation (using the algorithm from PDE toolbox of MATLAB). We use this procedure in order to obtain a correct computation of the initial particle volumes $V_{i}$ required by the scheme [22]. The SPH-MOOD-MLS scheme with cubic Taylor reconstruction and Rusanov flux is used for this example.

For the first blast wave problem (BWP1) case, the initial condition is

$$
(\rho, u, v, p)= \begin{cases}(1,0,0,1), & \text { if }|r| \leq 0.5 \\ (0.125,0,0,0.1), & \text { otherwise }\end{cases}
$$

The exact solution is constituted of cylindrical rarefaction, contact and shock waves.

Fig. 12, left column, displays the density and pressure approximations obtained at the final time, $t=0.2$. We observe a very good agreement with the reference solution, obtained with the NUMERICA library [41]. As in the one-dimensional case, we note a remarkable accuracy improvement of the approximation calculated with the SPH-MOOD-MLS method in comparison with the SPH-ALE base scheme. The solution is free from spurious oscillations and preserves the radial symmetry.

We perform another simulation using a random initial particle distribution (Fig. 11, bottom) initial distribution of particles. The numerical approximation is displayed in Fig. 12 right panel and the curves present a good agreement with the reference solution. 

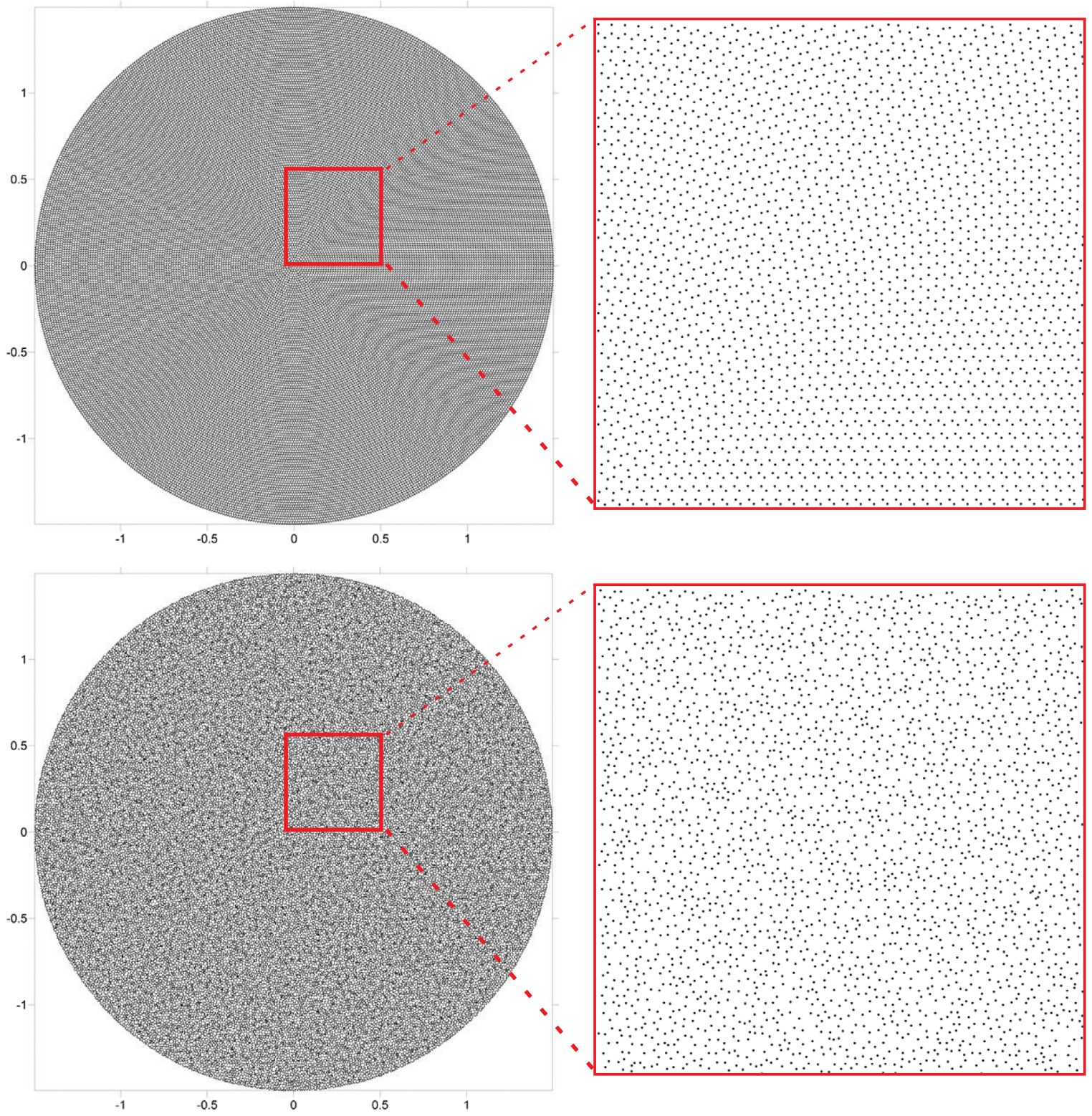

Fig. 11. Particle distributions used for 2D blast wave problems. Structured distribution (top) and unstructured distribution (bottom).

We plot in Fig. 13 the density, velocity and pressure for all particles as a function of particle radius in the structured case (top panels) and unstructured one (bottom panels). In the structured case, the particles maintain the symmetry of the solution thus the representation of the full set of particles as a one-dimensional plot (by rotation invariance) is valid. In the unstructured case, the symmetry is not as well reproduced as in the uniform case, in particular the velocity norm suffers an important deviation with respect to the other variables, density and pressure being less dispersed. We emphasize that the SPH-MOOD-MLS scheme is genuinely able to maintain cylindrical symmetry in presence of non-regular flow without spurious numerical effects.

For the second blast wave problem (BWP2) case, we set the following initial condition

$$
(\rho, u, v, p)= \begin{cases}(1,0,0,2), & \text { if }|r| \leq 0.5 \\ (1,0,0,1), & \text { otherwise }\end{cases}
$$



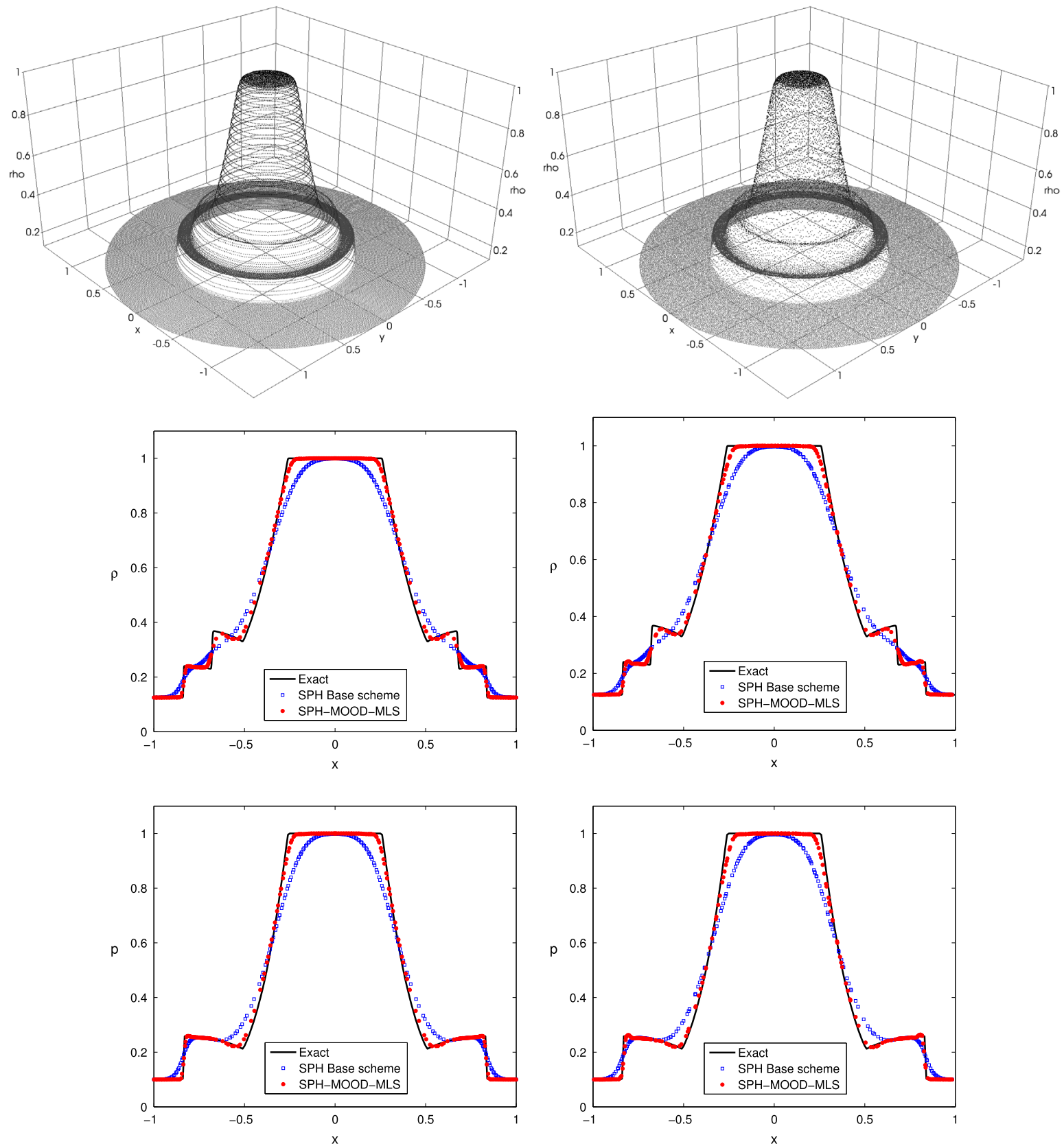

Fig. 12. Two dimensional blast wave BWP1 problem. On the left we plot the density results at $t=0.2$ obtained using a structured grid and on the right, the solution using an unstructured grid. We also plot the density (middle) and the pressure (bottom) results for a cut at $y=0$ using the novel SPH-MOOD-MLS scheme (filled circles) compared with the SPH base code (empty squares) and the reference solution (single line).

Fig. 14 shows the approximations for the density and pressure at final time $t=0.2$. As for the BWP1 case, the results calculated with the SPH-MOOD-MLS method are clearly more accurate that the ones obtained with the base scheme. They show a very good agreement with the reference solution.

Fig. 15 is the counterpart of Fig. 13, but for the BWP2 problem, both in the structured (top panels) and unstructured (bottom panels) cases. From these results we conclude that, for the structured and unstructured initialization, the scheme can maintain the underlying symmetry of the problem. In other words. the particles are not dispersed, the particles' values (density, velocity and pressure) are rather well approximated as a function of their radius. 

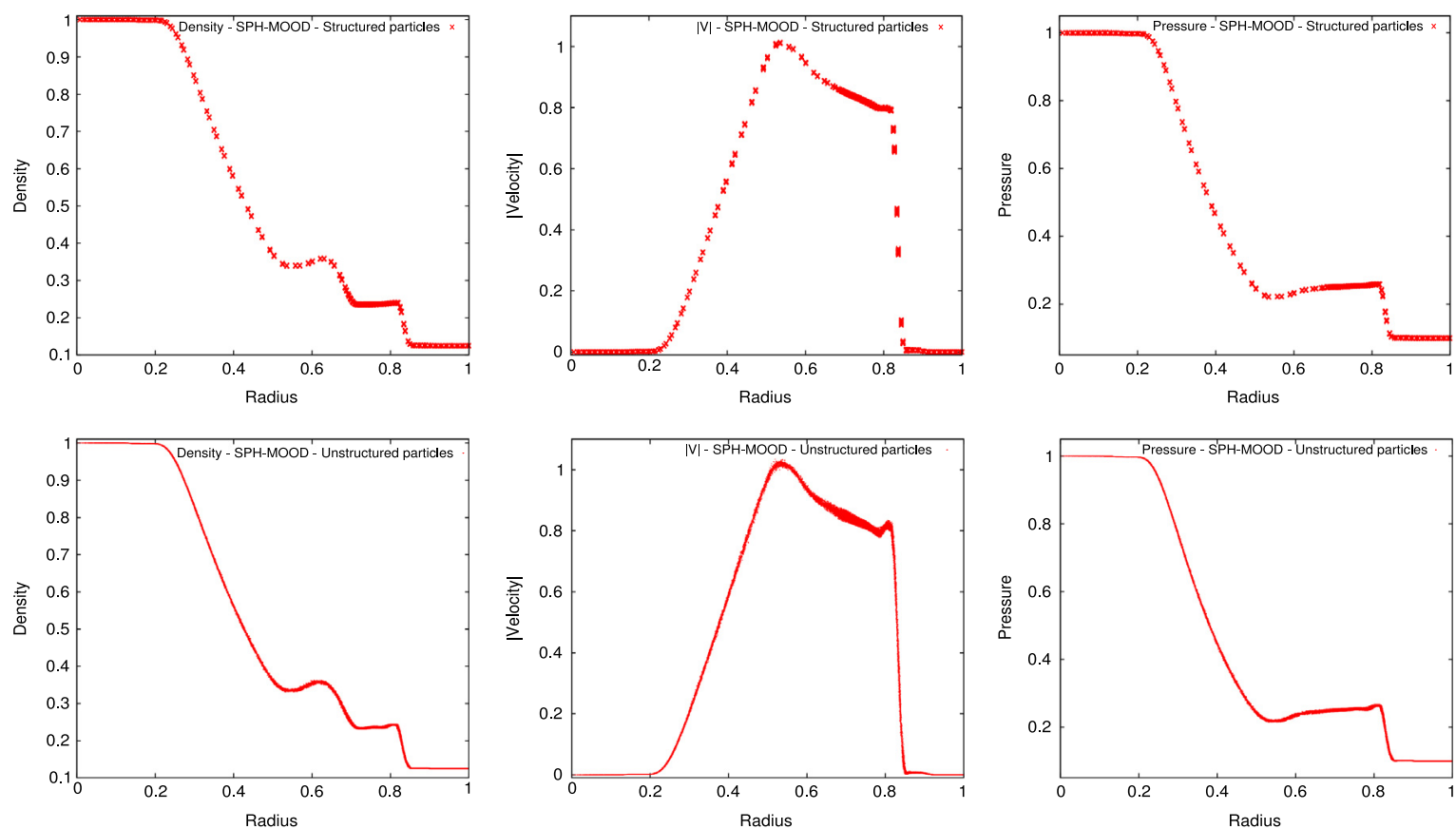

Fig. 13. Two dimensional blast wave BWP1 problem. Density (left panels), norm of the velocity (middle panels) and pressure (right panels) as a function of particle radius for all particles. Top panels present particles $(\times)$ which are initially as a regular polar mesh whereas bottom panels present the particles (.) which are randomly distributed (see Fig. 11).

\section{Conclusions}

In this work we have presented a new high-accurate SPH code. It is based on the use of Riemann solvers and Moving Least Squares for high accurate reconstruction of the left and right states of the Riemann problem. The stability of the numerical scheme is achieved by using the Multidimensional Optimal Order Detection (MOOD) paradigm. This a posteriori paradigm permits to stabilize the scheme without the call to classical limiting strategy such as slope limiter or artificial viscosity. The procedure only decreases the accuracy of the reconstructions when troubled cells are detected. Ultimately, locally to some troubles cells, the lowest possible accuracy of reconstruction can be reached, in other words the base SPH scheme is employed for those cells.

Our formulation obtains accurate and very promising results as demonstrated by several 1D and 2D test cases involving shock waves both with uniform or random initial distributions of particles. The MOOD paradigm greatly improves the accuracy on smooth flows when compared to the base scheme. Moreover, the proposed SPH-MOODMLS scheme maintains an essentially non-oscillatory behavior for non smooth flow without any spurious oscillations.

The numerical scheme presented here is formally second-order, due to the use of kernel approximations for the discretization of the divergence of the convective terms in the Euler equation [37]. This problem can however be overcome using MLS approximations to discretize these terms [24], and, in this context, the MOOD approach presented here can be applied without modification. Yet another possibility for reaching higher effective convergence orders is the extension of the MOOD paradigm to the choice of the kernel function. This possibility is currently being investigated by the authors.

\section{Acknowledgments}

This work has been partially supported by the Ministerio de Economía y Competitividad (grant \#DPI2015-68431R) of the Spanish Government and by the, Consellería de Cultura, Educación e Ordenación Universitaria of the Xunta de Galicia (grant \#GRC2014/039) cofinanced with FEDER funds, and the Universidade da Coruña. The 

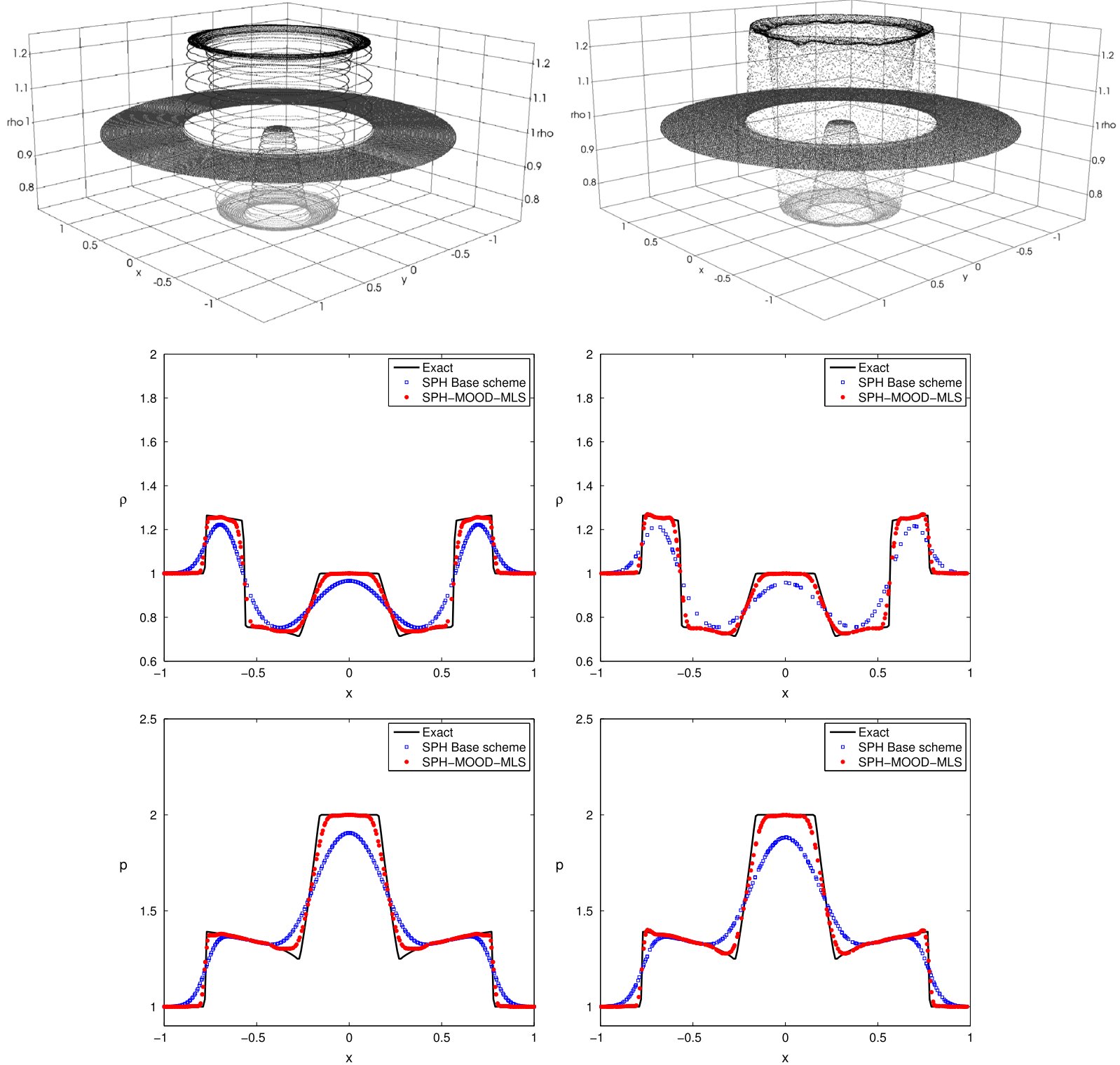

Fig. 14. Two dimensional blast wave BWP2 problem results at $t=0.2$. On the left we plot the density results obtained using a structured grid and on the right, the solution using an unstructured grid. We also plot the density (middle) and the pressure (bottom) results for a cut at $y=0$ using the novel SPH-MOOD-MLS scheme (filled circles) compared with the SPH base code (empty squares) and the reference solution (single line).

authors also acknowledge the Fundação para a Ciência e a Tecnologia for the funding of projects FCT-ANR/MATNAN/0122/2012 and UID/MAT/00013/2013.

The authors would like to thank the anonymous reviewers for their helpful and constructive comments that greatly contributed to improving the final version of the paper.

\section{Appendix. Derivation of the SPH-ALE scheme}

We recall the classical SPH formulation based on the convolution formulae. For a given set of approximation $\phi_{i} \approx \phi\left(x_{i}\right)$ of scalar values, we define an approximation of the gradient with the convolution 

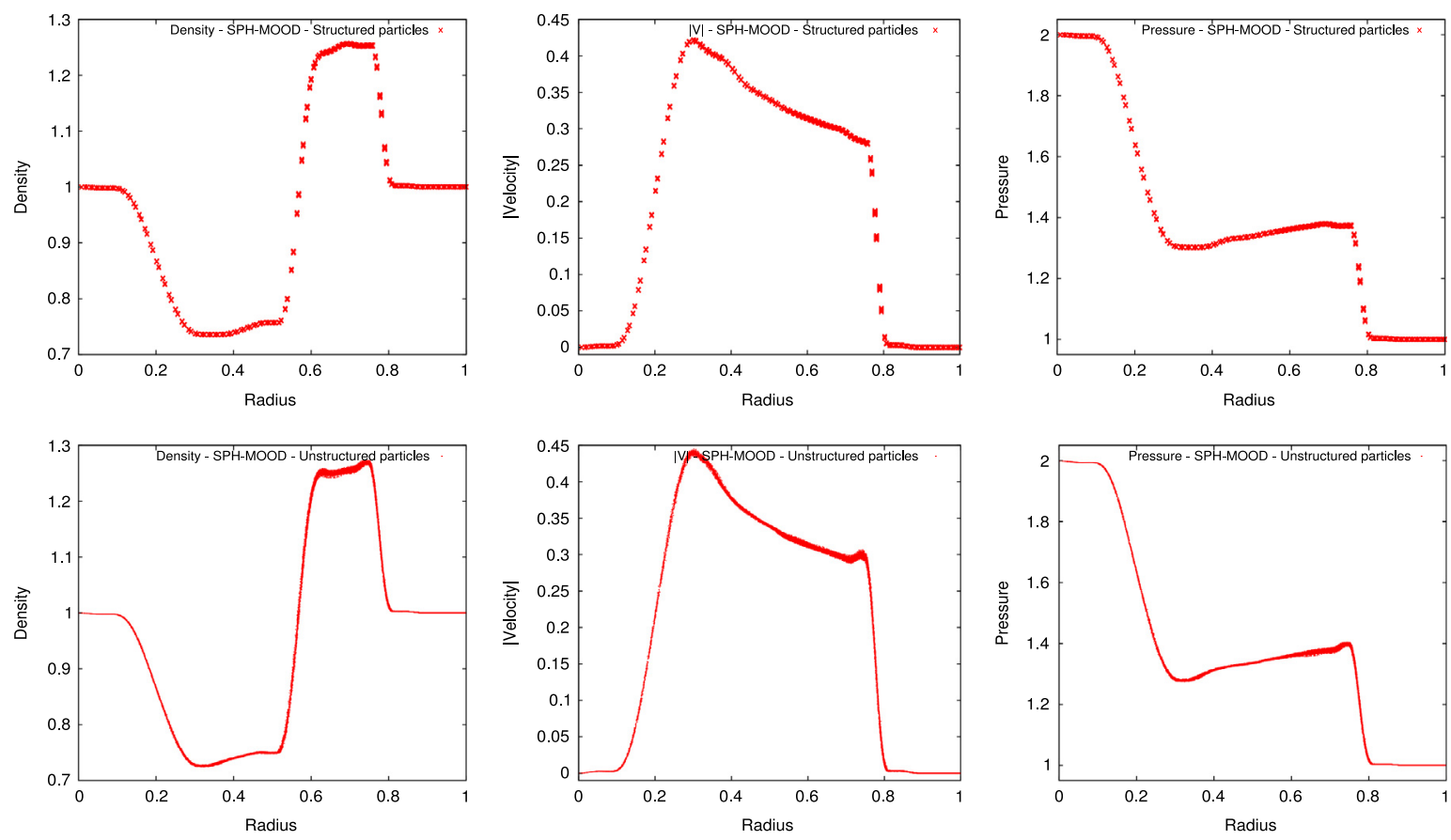

Fig. 15. Two dimensional blast wave BWP2 problem. Density (left panels), norm of the velocity (middle panels) and pressure (right panels) as a function of particle radius for all particles. Top panels present particles $(\times)$ which are initially as a regular polar mesh whereas bottom panels present the particles (.) which are randomly distributed (see Fig. 11).

$$
\nabla \phi_{i} \approx \sum_{j=1}^{n_{i}} V_{j} \phi_{j} \nabla W_{i j}
$$

which admits extensions for vectorial valued functions applying the operators component-wise. Assuming that a particle $i$ has a constant mass $m_{i}=V_{i} \rho_{i}$ in time, conservation yields

$$
\frac{d m_{i}}{d t}=0, \quad \frac{d\left(m_{i} u_{i}\right)}{d t}=m_{i} \nabla P_{i}, \quad \frac{d\left(m_{i} E_{i}\right)}{d t}=m_{i} u_{i} \cdot \nabla P_{i}
$$

we rewrite under the form

$$
\frac{d\left(V_{i} \rho_{i}\right)}{d t}=0, \quad \frac{d\left(V_{i} \rho_{i} u_{i}\right)}{d t}=V_{i} \rho_{i} \nabla P_{i}, \quad \frac{d\left(V_{i} \rho E_{i}\right)}{d t}=V_{i} \rho_{i} u_{i} \cdot \nabla P_{i} .
$$

On the other hand, using the relation $\nabla\left(\frac{P}{\rho}\right)=\frac{\nabla P}{\rho}-\frac{P \nabla \rho}{\rho^{2}}$, the gradient pressure becomes

$$
V_{i} \rho_{i} \nabla P_{i}=V_{i} \rho_{i}\left[\sum_{j=1}^{n_{i}} V_{j} \frac{P_{j}}{\rho_{j}} \nabla W_{i j}+\frac{P_{i}}{\rho_{i}} \sum_{j=1}^{n_{i}} V_{j} \rho_{j} \nabla W_{i j}\right]
$$

and system (26) turns to the symmetric formulation

$$
\begin{aligned}
& \frac{d}{d t}\left(V_{i} \rho_{i}\right)=0, \\
& \frac{d}{d t}\left(V_{i} \rho_{i} u_{i}\right)+V_{i} \sum_{j=1}^{n_{i}} V_{j}\left(\frac{\rho_{j}}{\rho_{i}} p_{i}+\frac{\rho_{i}}{\rho_{j}} p_{j}\right) \nabla W_{i j}=0, \\
& \frac{d}{d t}\left(V_{i} \rho_{i} E_{i}\right)+V_{i} \sum_{j=1}^{n_{i}} V_{j}\left(\frac{\rho_{j}}{\rho_{i}} p_{i} u_{i}+\frac{\rho_{i}}{\rho_{j}} u_{j} p_{j}\right) \nabla W_{i j}=0,
\end{aligned}
$$




$$
\frac{d x_{i}}{d t}=u_{i}
$$

Mass conservation in time provides an alternative formulation

$$
\begin{aligned}
& \rho_{i}=\sum_{j=1}^{n_{i}} m_{j} W_{i j}, \quad V_{i}=\frac{m_{i}}{\rho_{i}}, \\
& \frac{d u_{i}}{d t}+\sum_{j=1}^{n_{i}} m_{j}\left(\frac{p_{i}}{\rho_{i}^{2}}+\frac{p_{j}}{\rho_{j}^{2}}\right) \nabla W_{i j}=0, \\
& \frac{d E_{i}}{d t}+\sum_{j=1}^{n_{i}} m_{j}\left(\frac{p_{i}}{\rho_{i}^{2}} u_{j}+\frac{p_{j}}{\rho_{j}^{2}} u_{i}\right) \nabla W_{i j}=0 \\
& \frac{d x_{i}}{d t}=u_{i} .
\end{aligned}
$$

At last, for stability reasons, a pseudo-viscous pressure dissipation $\Pi_{i j}$ is added, leading to the standard SPH discretization for the Euler system

$$
\begin{aligned}
& \frac{d u_{i}}{d t}+\sum_{j=1}^{n_{i}} m_{j}\left(\frac{p_{i}}{\rho_{i}^{2}}+\frac{p_{j}}{\rho_{j}^{2}}+\Pi_{i j}\right) \nabla W_{i j}=0, \\
& \frac{d E_{i}}{d t}+\sum_{j=1}^{n_{i}} m_{j}\left(\frac{p_{i}}{\rho_{i}^{2}} u_{j}+\frac{p_{j}}{\rho_{j}^{2}} u_{i}+\Pi_{i j}\right) \nabla W_{i j}=0, \\
& \frac{d x_{i}}{d t}=u_{i} .
\end{aligned}
$$

We now turn to the SPH formulation based on the system

$$
L_{\boldsymbol{w}_{\text {frame }}} \boldsymbol{U}+\nabla \cdot\left(\mathcal{F}-\left(\boldsymbol{w}_{\text {frame }} \otimes \boldsymbol{U}\right)\right)=0
$$

with $L_{\boldsymbol{w}_{\text {frame }}} \boldsymbol{U}=\partial_{t} \boldsymbol{U}+\nabla \cdot\left(\boldsymbol{w}_{\text {frame }} \otimes \boldsymbol{U}\right)$ and $\boldsymbol{w}_{\text {frame }}$ the frame velocity. Following [11], we move the particles with the frame velocity and get

$$
\frac{d x_{i}}{d t}=\boldsymbol{w}_{\text {frame }}\left(x_{i}(t), t\right), \quad \frac{d V_{i}}{d t}=V_{i} \nabla \cdot \boldsymbol{w}_{\text {frame }}\left(x_{i}(t), t\right) .
$$

For any function $\phi(x, t)$, one has

$$
\frac{d\left(V_{i} \phi\left(x_{i}(t), t\right)\right)}{d t}=V_{i} L_{\boldsymbol{w}_{\text {frame }}} \phi\left(x_{i}(t), t\right) .
$$

Using such a relation for the conservative system provides

$$
\frac{d\left(V_{i} \boldsymbol{U}\left(x_{i}(t), t\right)\right)}{d t}+V_{i} \nabla \cdot\left(\mathcal{F}-\left(\boldsymbol{w}_{\text {frame }} \otimes \boldsymbol{U}\right)\right)\left(x_{i}(t), t\right)=0 .
$$

Applying the convolution to approximate de divergence provides

$$
\frac{d\left(V_{i} \boldsymbol{U}_{i}\right)}{d t}+V_{i} \sum_{j=1}^{n_{i}} V_{j}\left(\mathcal{F}_{j}-\left(\boldsymbol{w}_{\text {frame }} \otimes \boldsymbol{U}_{j}\right)\right) \nabla W_{i j}=0 .
$$

To enforce the condition that $\nabla 1=0$, we should have

$$
\sum_{j=1}^{n_{i}} V_{j}\left(\mathcal{F}_{i}-\left(\boldsymbol{w}_{\text {frame }} \otimes \boldsymbol{U}_{i}\right)\right) \nabla W_{i j}=\left(\mathcal{F}_{i}-\left(\boldsymbol{w}_{\text {frame }} \otimes \boldsymbol{U}_{i}\right)\right) \sum_{j=1}^{n_{i}} V_{j} \nabla W_{i j}=0,
$$


so we modify the approximation and provide a symmetric expression

$$
\frac{d\left(V_{i} \boldsymbol{U}_{i}\right)}{d t}+V_{i} \sum_{j=1}^{n_{i}} V_{j}\left\{\left(\mathcal{F}_{j}-\left(\boldsymbol{w}_{\text {frame }} \otimes \boldsymbol{U}_{j}\right)\right)+\left(\mathcal{F}_{i}-\left(\boldsymbol{w}_{\text {frame }} \otimes \boldsymbol{U}_{i}\right)\right)+\Pi_{i j}\right\} \nabla W_{i j}=0
$$

where $\Pi_{i j}$ is a diffusive term to stabilize the scheme. The case $\boldsymbol{w}_{\text {frame }}=\boldsymbol{u}$ corresponds to the Lagrangian formulation and we obtain a similar expression to the one derived from the classical SPH formulation (27)-(28).

Noticing that expression

$$
G_{i j}=\left\{\left(\mathcal{F}_{j}-\left(\boldsymbol{w}_{\text {frame }} \otimes \boldsymbol{U}_{j}\right)\right)+\left(\mathcal{F}_{i}-\left(\boldsymbol{w}_{\text {frame }} \otimes \boldsymbol{U}_{i}\right)\right)+\Pi_{i j}\right\} / 2
$$

corresponds to the centered flux with additional viscosity, it suggests that the SPH-ALE method takes a more generic form

$$
\frac{d\left(V_{i} \boldsymbol{U}_{i}\right)}{d t}+2 V_{i} \sum_{j=1}^{n_{i}} V_{j} G_{i j} \nabla W_{i j}=0
$$

where $G_{i j}$ stands for a numerical flux evaluated from particles $i$ to $j$ following the normalized direction $n_{i j}$. To compute an approximation $G_{i j}=G\left(\boldsymbol{U}_{i}, \boldsymbol{U}_{j}, n_{i j}\right)$ at the midpoint $x_{i j}$, we use first- or second-order numerical flux based on an approximation of the Riemann problem (Rusanov, HLLC).

Moreover, in order to completely define the system, it is required to define an equation for the definition of the weights. In [11,26] an SPH-like equation is used:

$$
\frac{d V_{i}}{d t}=-\sum_{j=1}^{n_{i}} V_{i} V_{j}\left(\boldsymbol{w}_{j}-\boldsymbol{w}_{i}\right) \cdot \nabla W_{i j}
$$

that can be written in terms of the velocity at the midpoint $\boldsymbol{w}_{i j}$ as

$$
\frac{d V_{i}}{d t}=-\sum_{j=1}^{n_{i}} V_{i} V_{j} 2\left(\boldsymbol{w}_{i j}-\boldsymbol{w}_{i}\right) \cdot \nabla W_{i j} .
$$

\section{References}

[1] L.B. Lucy, A numerical approach to the testing of the fission hypothesis, Astron. J. 82 (1977) 1013-1024.

[2] R.A. Gingold, J.J. Monaghan, Smoothed particle hydrodynamics-theory and application to non-spherical stars, Mon. Not. R. Astron. Soc. 181 (1977) 375-389.

[3] M.B. Liu, G.R. Liu, Smoothed Particle Hydrodynamics (SPH): an overview and recent developments, Arch. Comput. Methods Eng. 17 (2010) $25-76$.

[4] D.J. Price, Smoothed particle hydrodynamics and magnetohydrodynamics, J. Comput. Phys. 231 (3) (2012) 759-794.

[5] F.V. Sirotkin, J.J. Yoh, A smoothed particle hydrodynamics method with approximate Riemann solvers for simulation of strong explosions, Comput. \& Fluids 88 (2013) 418-429.

[6] K. Puri, P. Ramachandran, A comparison of SPH schemes for the compressible Euler equations, J. Comput. Phys. 256 (2014) $308-333$.

[7] S.-I. Inutsuka, Reformulation of smoothed particle hydrodynamics with Riemann solver, J. Comput. Phys. 179 (2002) $238-267$.

[8] D. Hietel, K. Steiner, J. Struckmeier, A finite-volume particle method for compressible flows, Math. Models Methods Appl. Sci. 10 (09) (2000) 1363-1382.

[9] T.Z. Ismagilov, Smooth volume integral conservation law and method for problems in Lagrangian coordinates, Comput. Math. Math. Phys. 46 (2006) 453-464.

[10] N. Quinlan, L. Lobovský, R.M. Nestor, Development of the meshless finite volume particle method with exact and efficient calculation of interparticle area, Comput. Phys. Comm. 185 (2014) 1554-1563.

[11] J.P. Vila, On particle weighted methods and smooth particle hydrodynamics, Math. Models Methods Appl. Sci. 9 (2) (1999) $161-209$.

[12] B. Ben Moussa, On the convergence of SPH method for scalar conservation laws with boundary conditions, Methods Appl. Anal. 13 (2006) 29-62.

[13] M. Junk, Do finite volume methods need a mesh? in: M. Griebel (Ed.), Meshfree Methods for Partial Differential Equations, Springer, 2003 , pp. 223-238.

[14] G.-A. Renaut, S. Aubert, J.-C. Marongiu, High order SPH-ALE method for hydraulic turbine simulations, in: 11th European Conference on Turbomachinery Fluid Dynamics and Thermodynamics, ETC 2015, Madrid, Spain, 2015.

[15] S. Clain, S. Diot, R. Loubère, A high-order finite volume method for systems of conservation laws- Multidimensional Optimal Order Detection (MOOD), J. Comput. Phys. 230 (2011) 4028-4050. 
[16] S. Diot, S. Clain, R. Loubère, Improved detection criteria for the Multidimensional Optimal Order Detection (MOOD) on unstructured meshes with very high-order polynomials, Comput. \& Fluids 64 (2012) 43-63.

[17] R. Loubère, M. Dumbser, Steven Diot, A new family of high order unstructured MOOD and ADER finite volume schemes for multidimensional systems of hyperbolic conservation laws, Commun. Comput. Phys. 16 (2014) 718-763.

[18] P. Lancaster, K. Salkauskas, Surfaces generated by moving least squares methods, Math. Comp. 37 (155) (1981) $141-178$.

[19] W.K. Liu, W. Hao, Y. Chen, S. Jun, J. Gosz, Multiresolution reproducing kernel particle methods, Comput. Mech. 20 (1997) $295-309$.

[20] L. Cueto-Felgueroso, I. Colominas, X. Nogueira, F. Navarrina, M. Casteleiro, Finite volume solvers and Moving Least-Squares approximations for the compressible Navier-Stokes equations on unstructured grids, Comput. Methods Appl. Mech. Engrg. 196 (2007) 4712-4736.

[21] X. Zhang, H. Tian, L. Kuo, W. Chen, A contact SPH method with high-order limiters for simulation of inviscid compressible flows, Commun. Comput. Phys. 14 (2) (2013) 425-442.

[22] D. Avesani, M. Dumbser, A. Bellin, A new class of moving-least-squares WENO-SPH schemes, J. Comput. Phys. 270 (2014) $278-299$.

[23] E. Gaburov, K. Nitadori, Astrophysical weighted particle magnetohydrodynamics, Mon. Not. R. Astron. Soc. 414 (2011) $129-154$.

[24] P.F. Hopkins, A new class of accurate, mesh-free hydrodynamic simulation methods, Mon. Not. R. Astron. Soc. 450 (1) (2015) 53-110.

[25] J. Bonet, T.S. Lok, Variational and momentum preservation aspects of smooth particle hydrodynamic formulations, Comput. Methods Appl. Mech. Engrg. 180 (1999) 97-115.

[26] M. Schaller, R.G. Bower, T. Theuns, On the use of particle based methods for cosmological hydrodynamical simulations, in: 8th International SPHERIC Workshop, Trondheim, Norway, June 4-6, 2013.

[27] D. Teleaga, J. Struckmeier, A finite-volume particle method for conservation laws on moving domains, Internat. J. Numer. Methods Fluids 58 (2008) 945-967.

[28] V.V. Rusanov, The calculation of the interaction of non-stationary shock waves and obstacles, USSR Comput. Math. Math. Phys. 1 (2) (1962) 304-320.

[29] G.R. Liu, M.B. Liu, Smoothed Particle Hydrodynamics. A Meshfree Particle Method, World Scientific Publishing, Singapore, 2003.

[30] G.R. Liu, Y.T. Gu, A point interpolation method, in: Proc 4th Asia-Pacific Conference on Computational Mechanics, Singapore, 1999, pp. 1009-1014.

[31] G.R. Liu, Y.T. Gu, K.Y. Dai, Assessment and applications of point interpolation methods for computational mechanics, Internat. J. Numer. Methods Engrg. 59 (2004) 1373-1397.

[32] T. Most, C. Bucher, New concepts for moving least squares: An interpolation non-singular weighting function and weighted nodal least squares, Eng. Anal, Bound. Elem. 32 (2008) 461-470.

[33] H. Wendland, Piecewise polynomial, positive definite and compactly supported radial functions of minimal degree, Adv. Comput. Math. 4 (1995) 389-396.

[34] S. Rosswog, Boosting the accuracy of SPH techniques: Newtonian and special-relativistic tests, Mon. Not. R. Astron. Soc. 448 (2015) 3628-3664.

[35] W. Benz, Smooth particle hydrodynamics: A review, in: J.R. Buchler (Ed.), The Numerical Modelling of Nonlinear Stellar Pulsations, in: NATO ASI Series, vol. 302, 1990, pp. 269-288.

[36] G.A. Dilts, Moving-least-squares-particle hydrodynamics. Consistency and stability, Internat. J. Numer. Methods Engrg. 44 (1999) 1115-1155.

[37] J.J. Monaghan, H. Pongracic, Artificial viscosity for particle methods, Appl. Numer. Math. 1 (1985) 187-194.

[38] S. Khelladi, X. Nogueira, F. Bakir, I. Colominas, Toward a higher order unsteady finite volume solver based on reproducing kernel methods, Comput. Methods Appl. Mech. Engrg. 200 (2011) 2348-2362.

[39] C.W. Shu, S. Osher, Efficient implementation of essentially non-oscillatory shock-capturing schemes, J. Comput. Phys. 77 (1988) $439-471$.

[40] G. Chiocchia, Exact Solutions to Transonic and Supersonic Flows, Technical Report AR-211, 1985.

[41] E.F. Toro, Riemann Solvers and Numerical Methods for Fluid Dynamics: A Practical introduction, Springer, ISBN: $9783540616764,1997$.

[42] H. Luo, J.D. Baum, R. Lohner, On the computation of multi-material flows using ALE formulation, J. Comput. Phys. 194 (1) (2004) $304-328$.

[43] G.A. Sod, A survey of several finite difference methods for systems of hyperbolic conservation laws, J. Comput. Phys. 27 (1978) 1-31. 